\title{
Coupled analysis of footbridge-pedestrian dynamic interaction
}

\author{
Maria Gabriella Mulas*, Eleonora Lai, Giulia Lastrico \\ Department of Civil and Environmental Engineering (DICA), Politecnico di Milano, Milano, Italy
}

ABSTRACT

In this work, an analytical formulation for the vibration response of a bridge due to walking pedestrians is proposed to the aim of modelling the human-structure interaction (HSI) in the vertical direction. Bridge and pedestrians are described as mechanical systems having a finite number of degrees of freedom (DOFs). A new single DOF model of a bipedal pedestrian is proposed, that reproduces the alternation of single and double support phases of human gait and the related ground reaction forces. The finite element method is adopted to model the 3D geometry of the bridge. The coupled equations of motion are derived based on the key assumption that contact points between the pedestrians and the bridge deck are massless. However, the structural matrices of the coupled system are time varying due to the pedestrian motion along the bridge. An uncoupled solution strategy is devised to reduce the computational burden, allowing for the separate integration of the bridge and the pedestrian sub-systems. The coupled formulation is uncoupled and associated with an iterative procedure that restores compatibility and equilibrium at contact points. The pedestrian model and the analytical procedure are implemented in a research code where input data are the bridge structural matrices computed with a commercial FE code. The modelling and analysis procedure is applied to a case study, a lively footbridge in Seriate, Italy. A first validation of the code is obtained by comparison with a closed form solution for a $1 \mathrm{D}$ beam. For the loading scenarios analyzed here, a maximum of two iterations per step are necessary to achieve con-vergence within a prescribed tolerance. Loading scenarios encompassing groups of pedestrians in different transverse positions highlight the importance of the 3D bridge modelling. The comparison with a few experi-mental results clarifies the role of the modelling assumptions. Conclusions discuss novelties, advantages, limits and future developments of the proposed approach.

\section{Introduction}

In civil engineering dynamics, human-induced vibrations have become a considerable serviceability issue due to the strong trend towards the design of light and slender structures, such as modern footbridges [1-3]. These structural systems often show natural frequencies in the range typical of human activities such as walking, running, bouncing, etc., so that the design requires careful attention to vibration levels [4]. At the design stage, the fulfilment of serviceability prescriptions requires a due consideration of some key aspects [5]. These include expected loading scenarios and dynamic properties of the structures, as well as the accuracy of the models representing dynamic loading and human response to vibration [6].

During footbridge vibration, some form of human-structure interaction (HSI) occurs [7]. HSI is a complex phenomenon in which pedestrians and footbridge interact as coupled mechanical systems in two possible ways: changes in the structural properties lead to changes in the humans' walking and vice versa $[8,9]$. Structural vibration can affect the human gait and consequently the forces induced by human occupants that, in turn, can change the bridge response [10]. This phenomenon is well documented for lateral vibration only $[2,11]$. In vertical vibration, the structural response due to pedestrians' motion cannot be predicted from the properties of the empty structure loaded by the pedestrian's forces. Thus, accounting for HSI is fundamental to predict a reliable dynamic response [6].

A large research effort performed over the last 15 years led to the development of human models and analytical procedures able to determine the footbridge response [12]. In a recent work, Caprani \& Ahmadi [13] illustrated different literature approaches for modelling both structures and pedestrians. For structures, either a formulation in modal coordinates or the Finite Element (FE) method are used. For pedestrians, dynamic travelling forces, mechanical systems, or a combination of both, are proposed. However, dynamic analyses using force models cannot treat the dynamic effects of the mechanical human body and the consequent HSI [14]. A force model describes only ground reaction forces (GRFs) applied by a pedestrian [15], leading to a

\footnotetext{
* Corresponding author.

E-mail addresses: mariagabriella.mulas@polimi.it (M.G. Mulas), eleonora.lai@arup.com (E. Lai), giulia.lastrico@mail.polimi.it (G. Lastrico).
} 
reduction in the accuracy of estimated bridge response [16]. On the contrary, a mechanical system also represents the mass, stiffness and damping of the pedestrian, all of which can combine with the structural properties [17].

To represent the human body, some authors have proposed simple mechanical systems, in which a complete representation of the human gait is not attempted (Zhang et al. [6,8], Shahabpoor et al. [16], Da Silva \& Pimentel [18,19], Van Nimmen et al. [20], Venuti et al. [21], Caprani et al. [13]). Other researchers adopted stiff or compliant bi-pedal models to simulate the leg-switching behavior of walking (Bocian et al. [22], Geyer et al. [23], Kim \& Park [24], Qin et al. [25], Whit-tington \& Thelen [26]).

Caprani \& Ahmadi [13] underlined that most of these studies do not present in detail the analytical formulation of the proposed models. In particular, they pointed out that (a) there is a need to provide the detailed formulation of HSI models, particularly under moving crowd scenarios; (b) presently, the models for HSI proposed in the literature are often not complemented with efficient integration procedures for the dynamic equations of motion, which would benefit other re-searchers in the field. Furthermore, to the best of the authors' knowl-edge, the literature does not offer examples of full 3D modelling of footbridges within a HSI analysis. In fact, in several works the foot-bridge is modelled as a simply supported Euler-Bernoulli beam with uniform cross-section (Zhang et al. [6], Bocian et al. [22], Qin et al.[25], Gomez et al. [27], Caprani \& Ahmadi [13]). This approach cannot capture the plate behavior of the deck and the effect of coupled tor-sional-flexural natural modes, which could be excited by eccentric transits of pedestrians. In addition, a 1D-bridge model cannot treat loading scenarios having different spatial distributions in the transverse direction and social force models necessary to include the interaction among pedestrians (humanhuman interaction).

Since humans transmit a three-component contact force, HSI should be accounted for in the longitudinal, transversal and vertical direction. Even though, as a starting point, the focus of this work is restricted to vertical interaction, HSI remains a multifaceted problem. The high variability of pedestrians' motion, in terms of trajectory, intra-varia-bility and inter-variability, has to be considered when pursuing a comprehensive approach to HSI. The derivation of a complete analy-tical formulation to compute the bridge response to walking pedes-trians, still lacking as pointed out from the above considerations, can be proposed and its applicability investigated. Thus, it is necessary to in-vestigate whether the type of pedestrian's motion modifies the me-chanical principles governing the footbridge response. If this is not the case, the two aspects can be analyzed separately.

The derivation of the coupled equation of motion requires the description of the two systems and of their interaction. The bridge model should describe accurately mass and stiffness of the structure, crucial in determining the modal properties, and the effects arising from human activities, as underlined in the European Guideline [28]. To this aim, a 3D FE model of the structure is a straightforward option. The pedestrian mechanical model on one hand should describe the dynamic properties of the human body and, on the other hand, transfer forces to the bridge only through its legs. The interaction between the two systems requires the definition of the compatibility conditions at the interface. A perfect contact can be assumed as in the solution strategy previously adopted for the vehicle-bridge interaction (VBI) problem in [29], taking ad-vantage of analogies between VBI and HSI. The finite area and the mass of feet can be neglected. While the VBI assumptions on contact hold for the HSI as well, the continuous wheel movement is completely different from the human walking or running. Thus, the model must be able to simulate the human gait and the derivation of the coupled equation must account for the real human locomotion and for the simultaneous presence of many pedestrians on the bridge.

The aim of this work is to derive an analytical formulation and a numerical procedure for the HSI, restricted to vertical direction, accounting for the above assumptions. Both systems, bridge and pedestrian, are described by means of discretized linear models. For the former, a 3D FE mesh is developed, with the refinement usual in engineering practice. For the latter, a new bipedal spring-mass-damper (SMD) model that simulates the human gait cycle is proposed. The model transmits contact forces through compliant and damped legs. As a working assumption, a periodic nature of the steps is considered in its first implementation, even though the sequence of footfalls in the human walking is non-periodic [30,31]. The coupled equations of motion are derived under the assumption of perfect contact at massless contact points. A solution strategy overcoming the problems related to the timevarying nature of the coupled matrices is proposed to integrate separately the two systems. This simplifies the representation of the force transfer typical of human gait, characterized by alternate loading applied by the feet.

After the description of the new bipedal pedestrian model in Section 2 , Section 3 presents the equation of motion of the pedestrian-bridge coupled system. The equations are subsequently uncoupled and an iterative solution strategy is established in Section 4. The case study in Section 5 is a lively footbridge analyzed in a previous work [32]. Section 6 describes the main features of the research code where the uncoupled formulation is implemented and the results of the analyses. A code validation is performed on a 1D simply supported beam. The performance of the iterative procedure is assessed. Finally, both the numerical response due to pedestrians' different trajectories and a comparison with an experimental test are presented. Conclusions in Section 7 highlight the advantages and novelties of the proposed approach and discuss limits and future developments.

\section{Pedestrian model}

The model represents a pedestrian who moves with a known motion in the longitudinal direction, along the line at the intersection between his sagittal and frontal planes. Since there is not lateral motion in the pedestrians' transverse plane, it is not necessary to consider degrees of freedom (DOFs) in the horizontal plane. Rigid-body rotations are neglected. The model simulates the typical human gait characterized by a sequence of alternate phases, in which either only one foot (Single Support Phase, SSP) or both feet (Double Support Phase, DSP) are in contact with the ground (Fig. 1a) [33]. Hence, each model's foot transmits a vertical contact force $F_{i}$, where $i=1,2$ refers to the leading and the trailing leg, respectively. As a working assumption, intravariability is neglected, and the model follows a deterministic and periodic representation of the human gait. Thus, its steps repeat identically with a period $T_{e}$ equal to the sum of the duration times of SSP and DSP, $T_{s}$ and $T_{d}$ respectively, and both feet transmit the same pattern of force at each step. In the literature, the reference model for the description of the GRFs transmitted by each foot is the one by Li et al.[34]. The position of the single foot force $F_{i}(t)$ is constant in the interval $T_{e}$. Its typical shape is depicted in Fig. 1b. In [34], $F_{i}(t)$ is the sum of five harmonics, whose amplitudes $A_{n}$ (or $D L F$, dynamic load factor) act as multipliers of the pedestrian weight $G$ :

$F(t)=G \sum_{n=1}^{5} A_{n} \sin \left(\frac{\pi n}{T_{e}}\right) \quad 0 \leqslant t \leqslant T_{e}$

The coefficients $A_{n}$ depend explicitly on the step frequency $f_{s}$, the inverse of the duration $T_{s}$ of the SSP. From the statistical results of Ebrahimpour [35], an average value of 4.16 is assumed for the ratio of $T_{e}$ and $T_{d}$. Thus:

$T_{s}=\frac{1}{f_{s}}=T_{e}-T_{d}=T_{e}-\frac{1}{4.16} T_{e}=0.76 T_{e}$

The proposed SMD bipedal mechanical model (Fig. 2) accounts for the above assumptions. The single DOF (SDOF) system has a mass $M_{h}$, the total mass of the human being, and is connected to the ground through two vertical spring-damper legs, acting in parallel in the same vertical 


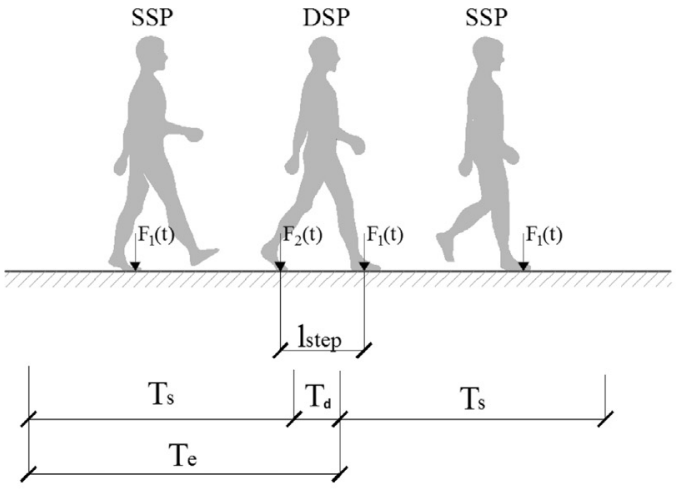

(a)

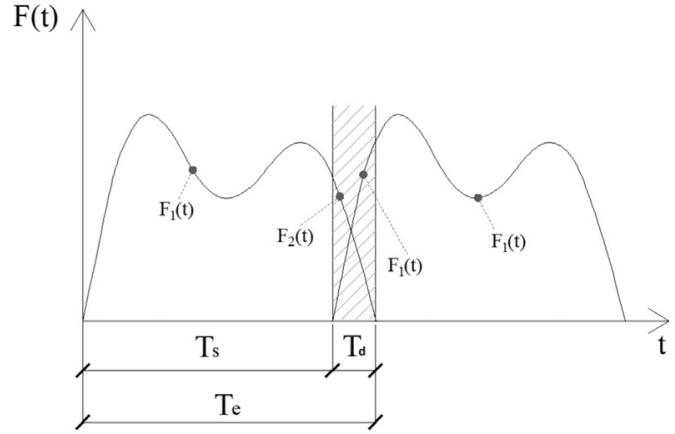

(b)

Fig. 1. Gait cycle: (a) sequence of single and double support phases; (b) vertical force transmitted by each foot during a cycle.

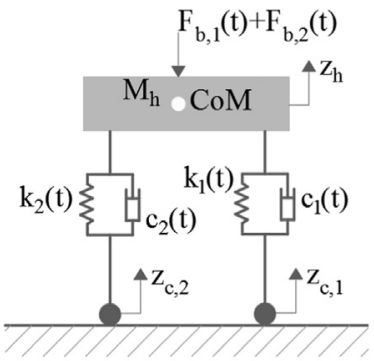

(a)

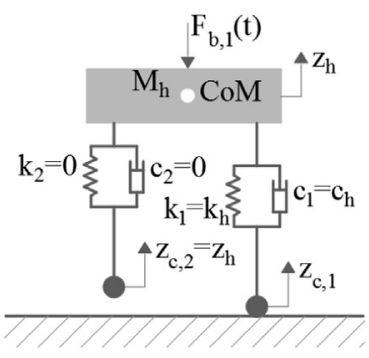

(b)
Fig. 2. Single DOF system modelling a bipedal walking pedestrian: (a) double and (b) single support phase.

plane. During DSP (Fig. 2a), both legs are in contact with the ground, at a distance $l_{\text {step }}$. During SSP (Fig. 2b), the leading leg is in contact while the trailing leg is "flying". With a well-consolidated assumption (e.g. Dougill et al. [36]), a perfect contact takes place at the foot-ground contact areas, modelled as massless points. During SSP, the stiffness and damping coefficients $k_{i}$ and $c_{i}$ of legs are constant and equal to $k_{h}$ and $c_{h}$ respectively. During DSP, the force of the leading leg decreases to zero, while the trailing leg transmits a force increasing from zero (Fig. 1b). A variation of the legs parameters can simulate this phenomenon. In this work, also to retain a linear behavior of the model, the stiffness and damping coefficients of each leg vary during DSP but their sum, $k_{h}$ and $c_{h}$ respectively, remains constant. The leg coefficients follow the same time-variation:

$k_{i}(t)=\alpha_{i}(t) k_{h} \quad c_{i}(t)=\alpha_{i}(t) c_{h} \quad i=1,2$

To make the variation of $\alpha_{1}(t)$ and $\alpha_{2}(t)$ independent of $T_{d}$, a non-dimensional time variable $\bar{t}$ is defined over DSP:

$\bar{t}=\frac{t-T_{S}}{T_{d}} \quad 0 \leqslant \bar{t} \leqslant 1$

As a first approximation, coefficients vary linearly. Since their sum remains constant, the functions $\alpha_{1}$ and $\alpha_{2}$ (Fig. 3a) must satisfy the conditions:

$\alpha_{1}(\bar{t})+\alpha_{2}(\bar{t})=1 \quad \alpha_{1}(0)=1 \quad \alpha_{1}(1)=0$

The model proposed by Kim and Park [24] provides the values of the model leg parameters as a function of the walking speed (units $\mathrm{m} / \mathrm{s}$ ) $v=l_{\text {step }} \times f_{s}$ [37]. The spring stiffness $K$ and damping ratio $\zeta$, normalized to the weight to height ratio, are computed as:

$K=30.16 v+14.93$

$\zeta=0.048 v-0.026$

The equation of motion for the pedestrian model is written with respect to the Lagrangian coordinate $z_{h}$ (Fig. 2), representing the absolute vertical displacement of the pedestrian centre of mass (CoM). Consistently, the vertical resultant of contact forces acts on the CoM without eccentricity. In the longitudinal direction, the position of the foot in contact does not vary in each support phase. In the vertical di-rection, perfect contact constrains the contact points to move as the surface the pedestrian is walking on. Thus, a further coordinate is in-troduced for each foot at contact points, $z_{c, 1}$ and $z_{c, 2}$ respectively (Fig. 2), which vanishes in case of a rigid surface. During SSP, the "flying foot" of the trailing leg is linked rigidly to the CoM and its motion is equal to $z_{h}$.

In the real case, the body metabolism (the human "internal engine") provides the energy necessary for walking and transmitting contact forces. In the model, an equivalent external "biomechanical" force $F_{b}$ acting on the CoM introduces energy. This approach differs from that in [36], aiming to the simulation of bobbing, where the driving force is an internal one. In fact, here the aim is to simulate the vertical oscillations (Fig. 2) loading the "legs" of the walking model, and the Lagrangian coordinate is an absolute one. $F_{b}$ is an equivalent or "model consistent" force since, when applied to the SMD system walking on a rigid surface, delivers the single foot force in Eq. (1) as output contact force. Hence, $F_{b}$ is computed numerically from the GRF through an inverse analysis performed in the frequency domain. To this aim the complex frequency response function (FRF) relating the (unknown) biomechanical force $F_{b}(t)$ and the (known) ground reaction force $F(t)$ in (1) can be first expressed. The FRF can be written as the sum of the elastic and viscous forces transmitted to the ground by the oscillator undergoing steadystate motion under a unit harmonic force:

$H_{F_{b} F}(f)=k_{h} H(f)+i c_{h} 2 \pi f H(f)$

In (8) $i$ is the imaginary unit and $f$ denotes frequency. $H(f)$ is the usual FRF relating the displacement of a SDOF system to the applied dynamic force, i.e.:

$H(f)=\frac{1}{k_{h}} \cdot \frac{1}{1-\left(f / f_{h}\right)^{2}+i 2 \pi f\left(f / f_{h}\right)}$

In (9) $f_{h}$ is the natural frequency of the pedestrian model. Once the FRF (8) is defined, the Fourier Transform (FT) $\widetilde{F}_{b}(t)$ of the biomechanical force is obtained from the FT of the ground reaction force $\widetilde{F}(f)$ as $\widetilde{F}_{b}(t)=\widetilde{F}(f) / H_{F_{b} F}(f)$. Finally, the time-history of the biomechanical force is obtained by performing the inverse FT. As an example, $F_{b}(t)$ has been numerically computed according to the above-sketched procedure and by use of Discrete Fourier Transforms for the case of a pedestrian having a mass of $80 \mathrm{~kg}$, height of $1.80 \mathrm{~m}$ and travelling at $1.3 \mathrm{~m} / \mathrm{s}$. Fig. 3b depicts the input and output forces.

Finally, the equation of motion of the SMD model, accounting for the alternation between SSP and DSP governed by the time intervals $T_{e}$ and $T_{s}$, is written for both phases as: 


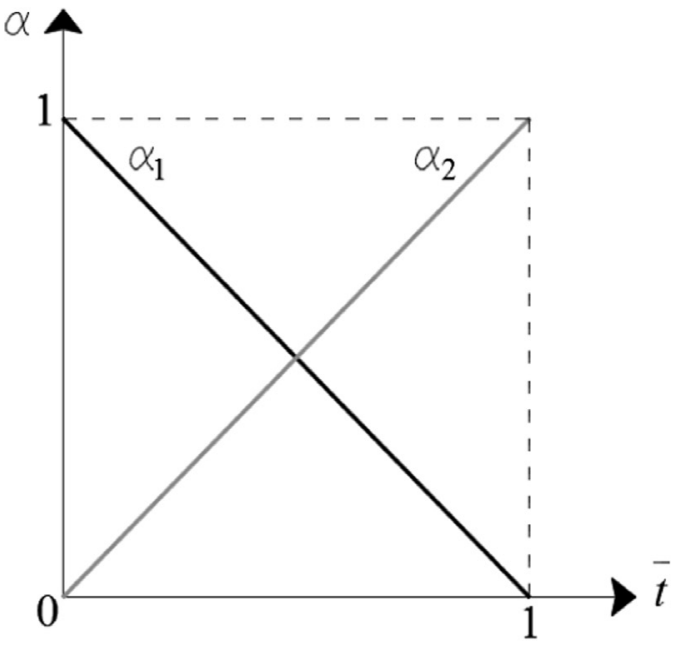

(a)

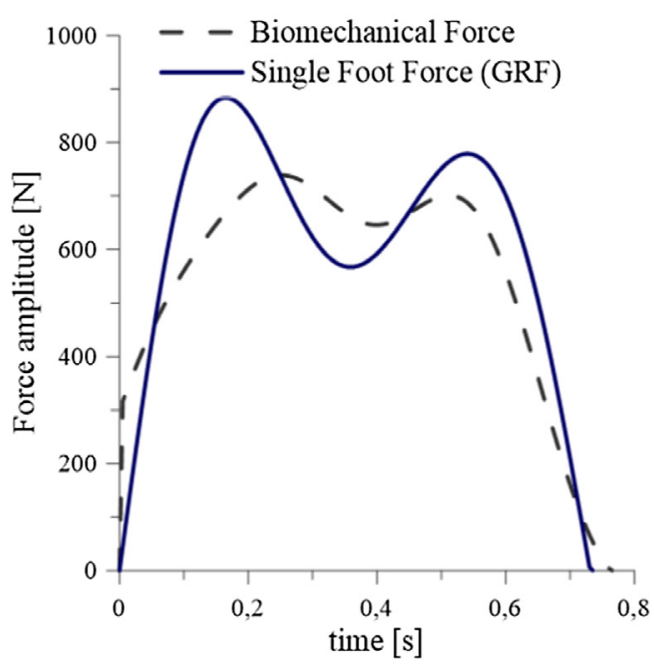

(b)

Fig. 3. Pedestrian model. (a) Functions $\alpha_{1}(\bar{t})$ and $\alpha_{2}(\bar{t})$. (b) Biomechanical force (input) and single foot force (output) for $f_{s}=1.8 \mathrm{~Hz}, v_{s}=1.3 \mathrm{~m} / \mathrm{s}$.

$$
\begin{aligned}
& M_{h} \ddot{z}_{h}+c_{h} \dot{z}_{h}+k_{h} z_{h} \\
& \quad= \begin{cases}F_{b, 1}(t)+c_{h} \dot{z}_{c, 1}+k_{h} z_{c, 1} & S S P \\
F_{b, 1}(t)+F_{b, 2}(t)+c_{1} \dot{z}_{c, 1}+k_{1} z_{c, 1}+c_{2} \dot{z}_{c, 2}+k_{2} z_{c, 2} & D S P\end{cases}
\end{aligned}
$$

In (10) only the right hand side (RHS) depends on the phase within the gait cycle, reflecting the linearity of the system. Two biomechanical forces, one for each leg, act during DSP (see also Fig. 2a). Given $z_{h}$, the equilibrium condition at the constrained DOFs provides the contact forces, computed as the opposite of the internal forces in legs:

$F_{c, 1}=c_{h}\left(\dot{z}_{h}-\dot{z}_{c, 1}\right)+k_{h}\left(z_{h}-z_{c, 1}\right) \quad S S P$

$F_{c, i}=c_{h}\left(\dot{z}_{h}-\dot{z}_{c, i}\right)+k_{h}\left(z_{h}-z_{c, i}\right) \quad D S P \quad i=1,2$

The forces defined in (11a) and (11b) are the whole contact forces. In this respect, this approach differs from the one in [38], where the contact force is fictitiously computed as the sum of the GRFs on a rigid surface and of a term coming from the interaction with the vibrating structure.

\section{Equations of motion for coupled system}

The derivation of the bridge-pedestrian coupled equation of motion follows the approach adopted on a previous work on vehicle-bridge interaction [29]. The derivation is based on the following assumptions: (a) the bridge is modelled with the FE method, as usual in the engineering practice; (b) the pedestrian is modelled as a bipedal SMD system and walks with a given horizontal velocity $v_{h}$, independent of his vertical motion; (c) contact forces are vertical; (d) perfect contact takes place at massless contact points.

Without loss of generality, we assume that a rectangular grid represents the mesh of the bridge deck. Contact points can be everywhere on the deck, not necessarily coincident with mesh nodes. At the generic time instant $t$, each contact force directly loads the nodes of the crossed mesh element (Fig. 4a). If the trajectory coincides with a line of nodes, the loaded nodes are those immediately before and after the contact point (Fig. 4b). The vector $\boldsymbol{q}$ of the Lagrangian coordinates for the coupled bridge-pedestrian system lists the bridge coordinates $\widehat{\boldsymbol{q}_{\boldsymbol{b}}}$ and the pedestrian's one $\widehat{\boldsymbol{q}_{h}}$ :

$\boldsymbol{q}=\left[\begin{array}{l}\hat{\boldsymbol{q}}_{b} \\ \widehat{\boldsymbol{q}}_{\boldsymbol{h}}\end{array}\right]$

Taking into account the pedestrian's position, the bridge vector $\widehat{\boldsymbol{q}_{\boldsymbol{b}}}$ is further partitioned into $\boldsymbol{q}_{b}$ and $\boldsymbol{q}_{c}$. As schematically depicted in Fig. 4c, the latter lists the coordinates of the directly loaded nodes, the former covers the remaining nodes (and coordinates) of the bridge:

$\widehat{\boldsymbol{q}}_{b}=\left[\begin{array}{l}\boldsymbol{q}_{b} \\ \boldsymbol{q}_{c}\end{array}\right]$

The contact point on the bridge at the generic time $t$ is not a mesh node. Hence, its coordinates $\boldsymbol{q}_{\boldsymbol{c} b}$ (Fig. 4c) are not listed in $\hat{\boldsymbol{q}}_{\boldsymbol{b}}$. To solve the problem, they are related to the coordinates $\boldsymbol{q}_{\boldsymbol{c}}$ through a set of shape functions $\boldsymbol{N}(\boldsymbol{x}(t))$, where $\boldsymbol{x}(t)$ is the position vector of the pedestrian:

$q_{c b}=N(x(t)) q_{c}$

Similar to the bridge vector, the pedestrian's sub-vector $\widehat{\boldsymbol{q}}_{\boldsymbol{h}}$ is partitioned into $\boldsymbol{q}_{\boldsymbol{c h}}$ and $\boldsymbol{q}_{\boldsymbol{h}}$. The former contains the constrained DOFs at contact points, the latter lists the remaining coordinates:

$\widehat{\boldsymbol{q}}_{\boldsymbol{h}}=\left[\begin{array}{c}\boldsymbol{q}_{c h} \\ \boldsymbol{q}_{h}\end{array}\right]$

For the SMD model in Section $2, \boldsymbol{q}_{c h}$ contains the coordinates $z_{c i}$ in (10), (11a) and (11b), $\boldsymbol{q}_{\boldsymbol{h}}$ the coordinate $z_{h}$. The assumption of perfect contact generates a compatibility condition between bridge and pedestrian:

$\boldsymbol{q}_{c h}=\boldsymbol{q}_{c b}$

Taking into account (14), Eq. (16) can be rewritten as:

$q_{c h}=N(x(t)) q_{c}$

The time derivative of (17) is necessary to compute the kinetic energy $\mathrm{T}$ and the dissipation function $D$ of the coupled system. Since the velocity $v_{c}$ of the contact points is null, the convective term vanishes in this case:

$\dot{q}_{c h}=\dot{q}_{c b}=N \dot{q}_{c}+N \dot{q}_{c}=N \dot{q}_{c}+N^{\prime} v_{c} q_{c}=N \dot{q}_{c}$

Taking into account (17), the sub-vector $\boldsymbol{q}_{\boldsymbol{c h}}$ disappears from the vector $\boldsymbol{q}$ in (12):

$[\boldsymbol{q}]=\left[\begin{array}{l}\widehat{\boldsymbol{q}}_{b} \\ \widehat{\boldsymbol{q}}_{\boldsymbol{h}}\end{array}\right]=\left[\begin{array}{l}\boldsymbol{q}_{b} \\ \boldsymbol{q}_{c} \\ \boldsymbol{q}_{h}\end{array}\right]$

The equations of motion of the coupled system are derived using the Lagrange equation:

$\frac{d}{d t}\left(\frac{\partial T(\dot{\boldsymbol{q}}, t)}{\partial \dot{\boldsymbol{q}}_{k}}\right)+\frac{\partial V(\boldsymbol{q}, t)}{\partial \boldsymbol{q}_{k}}+\frac{\partial D(\dot{\boldsymbol{q}}, t)}{\partial \dot{\boldsymbol{q}}_{k}}=\boldsymbol{Q}_{k}^{(n . c .)} \quad k=b, c, h$

In (20), the subscript " $k$ " spans bridge (" $b$ "), contact ("c") and human (" $h$ ") vector coordinates and $\boldsymbol{Q}_{k}{ }^{(\text {n.c.) }}$ is the vector of non-conservative 


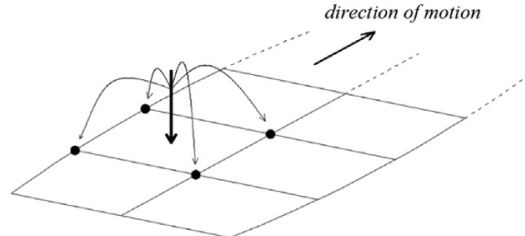

(a)

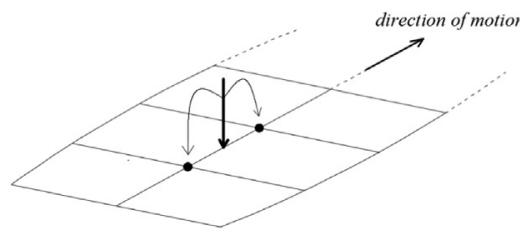

(b)

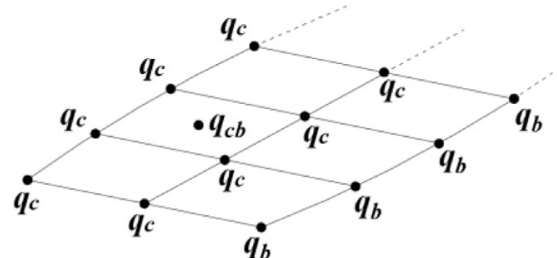

(c)

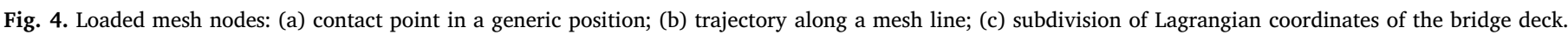
Labels $\boldsymbol{q}_{c} / \boldsymbol{q}_{b}$ denote nodes that will be directly/indirectly loaded during the entire pedestrian's transit.

forces. The contributions of both systems are summed to provide the kinetic energy $T$, the potential energy $V$, and the Rayleigh dissipation function $D$. Eqs. (12)-(20) are formally independent of the SMD model adopted.

With the meaning of subscripts introduced above, all the structural matrices of the two systems are partitioned as the corresponding vector of the coordinates. Since the contact points are massless and the pedestrian moves with a constant horizontal velocity $v_{h}$, taking into account (18) the kinetic energy of the coupled system is:

$$
\begin{aligned}
T= & T_{B}+T_{H}=\frac{1}{2}\left[\begin{array}{ll}
\dot{\boldsymbol{q}}_{\boldsymbol{b}}^{T} & \dot{\boldsymbol{q}}_{\boldsymbol{c}}^{T}
\end{array}\right]\left[\begin{array}{cc}
\boldsymbol{m}_{\boldsymbol{b}, \boldsymbol{b}} & \boldsymbol{m}_{\boldsymbol{b}, \boldsymbol{c}} \\
\boldsymbol{m}_{\boldsymbol{b}, \boldsymbol{c}}^{\boldsymbol{T}} & \boldsymbol{m}_{\boldsymbol{c}, \boldsymbol{c}}
\end{array}\right]\left[\begin{array}{c}
\dot{\boldsymbol{q}}_{\boldsymbol{b}} \\
\dot{\boldsymbol{q}}_{\boldsymbol{c}}
\end{array}\right] \\
& +\frac{1}{2}\left(\left[\begin{array}{ll}
\dot{\boldsymbol{q}}_{\boldsymbol{c}}^{\boldsymbol{T}} \boldsymbol{N}^{\boldsymbol{T}} & \dot{\boldsymbol{q}}_{\boldsymbol{h}}^{T}
\end{array}\right]\left[\begin{array}{cc}
\mathbf{0} & \mathbf{0} \\
\mathbf{0} & \boldsymbol{m}_{\boldsymbol{h}, \boldsymbol{h}}
\end{array}\right]\left[\begin{array}{c}
N \dot{\boldsymbol{q}}_{\boldsymbol{c}} \\
\dot{\boldsymbol{q}}_{\boldsymbol{h}}
\end{array}\right]+M_{h} v_{h}^{2}\right)
\end{aligned}
$$

In computing the potential energy, the contribution of the bridge static loads is accounted for through the vector $\boldsymbol{Q}_{\mathbf{0}}$. Taking into account (17) we obtain:

$$
\begin{aligned}
V= & V_{B}+V_{H}=\frac{1}{2}\left[\begin{array}{ll}
\boldsymbol{q}_{b}^{T} & \boldsymbol{q}_{c}^{T}
\end{array}\right]\left[\begin{array}{ll}
\boldsymbol{k}_{b, b} & \boldsymbol{k}_{b, c} \\
\boldsymbol{k}_{b, c}^{T} & \boldsymbol{k}_{c, c}
\end{array}\right]\left[\begin{array}{l}
\boldsymbol{q}_{b} \\
\boldsymbol{q}_{c}
\end{array}\right]-\left[\begin{array}{ll}
\boldsymbol{Q}_{0 b}^{T} & Q_{0 c}^{T}
\end{array}\right]\left[\begin{array}{l}
\boldsymbol{q}_{b} \\
\boldsymbol{q}_{c}
\end{array}\right] \\
& +\frac{1}{2}\left[\begin{array}{ll}
\boldsymbol{q}_{c}^{T} \boldsymbol{N}^{T} & \boldsymbol{q}_{h}^{T}
\end{array}\right]\left[\begin{array}{ll}
\boldsymbol{k}_{c h, c h} & \boldsymbol{k}_{c h, h} \\
\boldsymbol{k}_{c h, h}^{T} & \boldsymbol{k}_{h, h}
\end{array}\right]\left[\begin{array}{c}
\boldsymbol{N} \boldsymbol{q}_{c} \\
\boldsymbol{q}_{h}
\end{array}\right]
\end{aligned}
$$

Taking into account (18), the function $D$ is:

$$
\begin{aligned}
& D=D_{B}+D_{H}=\frac{1}{2}\left[\begin{array}{ll}
\dot{\boldsymbol{q}}_{\boldsymbol{b}}^{T} & \dot{\boldsymbol{q}}_{\boldsymbol{c}}^{T}
\end{array}\right]\left[\begin{array}{ll}
\boldsymbol{c}_{\boldsymbol{b}, \boldsymbol{b}} & \boldsymbol{c}_{\boldsymbol{b}, \boldsymbol{c}} \\
\boldsymbol{c}_{\boldsymbol{b}, \boldsymbol{c}}^{\boldsymbol{T}} & \boldsymbol{c}_{\boldsymbol{c}, \boldsymbol{c}}
\end{array}\right]\left[\begin{array}{l}
\dot{\boldsymbol{q}}_{\boldsymbol{b}} \\
\dot{\boldsymbol{q}}_{\boldsymbol{c}}
\end{array}\right] \\
& +\frac{1}{2}\left[\begin{array}{ll}
\dot{\boldsymbol{q}}_{c}^{T} \boldsymbol{N}^{T} & \dot{\boldsymbol{q}}_{h}^{T}
\end{array}\right]\left[\begin{array}{cc}
c_{c h, c h} & c_{c h, h} \\
c_{c h, h}^{T} & c_{h, h}
\end{array}\right]\left[\begin{array}{c}
N \dot{\boldsymbol{q}}_{c} \\
\dot{\boldsymbol{q}}_{h}
\end{array}\right]
\end{aligned}
$$

The only non-zero term in the vector $\boldsymbol{Q}^{(\text {n.c.) }}$ is given by the equivalent biomechanical forces:

$Q^{(\text {n.c. })}=\left[\begin{array}{c}0 \\ 0 \\ F_{b}\end{array}\right]$

By standard derivations, the equations of motion are obtained:

$$
\begin{gathered}
{\left[\begin{array}{ccc}
m_{b, b} & m_{b, c} & 0 \\
m_{b, c}^{T} & m_{c, c} & 0 \\
0 & 0 & m_{h, h}
\end{array}\right]\left[\begin{array}{l}
\ddot{q}_{b} \\
\ddot{q}_{c} \\
\ddot{q}_{h}
\end{array}\right]+\left[\begin{array}{ccc}
c_{b, b} & c_{b, c} & 0 \\
c_{b, c}^{T} & c_{c, c}+N^{T} c_{c h, c h} N & N^{T} c_{c h, h} \\
0 & c_{c h, h}^{T} N & c_{h, h}
\end{array}\right]\left[\begin{array}{c}
\dot{q}_{b} \\
\dot{q}_{c} \\
\dot{q}_{h}
\end{array}\right]+} \\
+\left[\begin{array}{ccc}
k_{b, b} & k_{b, c} & 0 \\
k_{b, c}^{T} & k_{c, c}+N^{T} k_{c h, c h} N & N^{T} k_{c h, h} \\
0 & k_{c h, h}^{T} N & k_{h, h}
\end{array}\right]\left[\begin{array}{l}
q_{b} \\
q_{c} \\
q_{h}
\end{array}\right]=\left[\begin{array}{c}
Q_{0 b} \\
Q_{0 c} \\
0
\end{array}\right]+\left[\begin{array}{c}
0 \\
0 \\
F_{b}
\end{array}\right]
\end{gathered}
$$

The mass matrix $\boldsymbol{M}$ in (25) is uncoupled and time-invariant due to the assumption of massless contact points. The damping matrix $\boldsymbol{C}$ and stiffness matrix $\boldsymbol{K}$ are symmetric, due to the null value of the convective term in (18), and show the same pattern. The coupling between the coordinates $\boldsymbol{q}_{c}$ and $\boldsymbol{q}_{h}$ is due only to terms related to contact points that contain the time-dependent shape function matrix $N$. When $n$ pedestrians are on the bridge, the diagonal matrices $\boldsymbol{m}_{\boldsymbol{h}, \boldsymbol{h}}, \boldsymbol{c}_{\boldsymbol{h}, \boldsymbol{h}}$ and $\boldsymbol{k}_{\boldsymbol{h}, \boldsymbol{h}}$ have dimensions $(n \times n)$. The dimensions of diagonal matrices $\boldsymbol{c}_{\boldsymbol{c h}, \boldsymbol{c h}}$ and $\boldsymbol{k}_{\boldsymbol{c h}, \boldsymbol{c h}}$ for a single pedestrian are $(1 \times 1)$ and $(2 \times 2)$ during SSP and DSP, respectively. For obvious reasons, each contact point belongs to one and only one pedestrian. No direct coupling takes place among pedestrians, apart from the indirect effect due to the bridge response.

To analyze the effect of the bridge static deflection in (25), the vector $\boldsymbol{q}$ is decomposed into the sum of the bridge static deflection $\boldsymbol{q}_{0}$ and of the dynamic coupled response of both systems $\boldsymbol{q}_{\boldsymbol{d}}$ :

$[\boldsymbol{q}]=\left[\begin{array}{c}\boldsymbol{q}_{b} \\ \boldsymbol{q}_{c} \\ \boldsymbol{q}_{h}\end{array}\right]=\left[\begin{array}{c}\boldsymbol{q}_{0 b} \\ \boldsymbol{q}_{0 c} \\ 0\end{array}\right]+\left[\begin{array}{c}\boldsymbol{q}_{d b} \\ \boldsymbol{q}_{d c} \\ \boldsymbol{q}_{h}\end{array}\right]=\boldsymbol{q}_{0}+\boldsymbol{q}_{d}$

When (26) is inserted in (25), the vector of static forces $\boldsymbol{Q}_{0}$ at the RHS disappears since the static equilibrium condition of the bridge $\boldsymbol{K}_{\boldsymbol{b}} \boldsymbol{q}_{0}=\boldsymbol{Q}_{0}$ holds. The structural matrices in (25) are not affected by the decomposition (26) and the equations of motion can be rewritten in a compact form as:

$M \ddot{q}_{d}+C \dot{q}_{d}+K q_{d}=Q^{(n . c .)}-Q_{0 c}$

where:

$Q_{0 c}(t)=\left[\begin{array}{c}0 \\ N^{T} k_{c h, c h} N q_{0 c} \\ k_{c h, h}^{T} N q_{0 c}\end{array}\right]$

\section{Uncoupled formulation and iterative procedure}

The Eq. (27) formally solves the problem of deriving the equations of motion of the coupled system. However, the stiffness and damping matrices in (25) are time dependent. This implies that, whenever a pedestrian's foot changes its position on the bridge, it is necessary to update the structural matrices and their topology and to triangularize the equivalent stiffness matrix adopted in the numerical integration. The solution strategy proposed here is based on a forced uncoupling, obtained by moving the coupling terms of (27) to the RHS [29]. The structural matrices become block diagonal: the first two rows refer to the bridge, the last one to the pedestrian. The equation of motion for the footbridge is written as:

$$
\begin{gathered}
{\left[\begin{array}{ll}
m_{b, b} & m_{b, c} \\
m_{b, c}^{T} & m_{c, c}
\end{array}\right]\left[\begin{array}{l}
\ddot{q}_{d b} \\
\ddot{q}_{d c}
\end{array}\right]+\left[\begin{array}{ll}
c_{b, b} & c_{b, c} \\
c_{b, c}^{T} & c_{c, c}
\end{array}\right]\left[\begin{array}{l}
\dot{q}_{d b} \\
\dot{q}_{d c}
\end{array}\right]+\left[\begin{array}{ll}
k_{b, b} & k_{b, c} \\
k_{b, c}^{T} & k_{c, c}
\end{array}\right]\left[\begin{array}{l}
q_{d b} \\
q_{d c}
\end{array}\right]=\left[\begin{array}{c}
0 \\
N^{T} f
\end{array}\right]} \\
=\left[\begin{array}{l}
0 \\
P
\end{array}\right]
\end{gathered}
$$

In (29) the vector $f$ contains the forces exerted by the pedestrian at contact points, transformed into proper nodal forces $\boldsymbol{P}$ when pre-multiplied by $\boldsymbol{N}^{T}$ :

$$
\begin{aligned}
f= & -\left(k_{c h, c h} N q_{d c}+k_{c h, h} q_{h}+c_{c h, c h} N \dot{q}_{d c}+c_{c h, h} \dot{q}_{h}\right. \\
& \left.+\left(k_{c h, c h} N+c_{c h, c h} N\right) q_{0 c}\right)
\end{aligned}
$$

The equation of motion for the pedestrian mechanical system is written as: 
$\boldsymbol{m}_{h, h} \ddot{\boldsymbol{q}}_{h}+c_{h, h} \dot{\boldsymbol{q}}_{h}+\boldsymbol{k}_{h h} \boldsymbol{q}_{h}=F_{b}+\boldsymbol{f}_{h}$

The vector $\boldsymbol{f}_{\boldsymbol{h}}$ in (31) contains the pedestrian's forcing term depending on its base motion:

$f_{h}=-\left(k_{c h, h}^{T} N q_{d c}+c_{c h, h}^{T} N \dot{q}_{d c}+k_{c h, h}^{T} N q_{0 c}\right)$

The uncoupled equations can be solved only through an iterative procedure. The iteration strategy named STS (Single Time Step) in [29] is adopted here, where iteration is performed at each time step, moving back and forth from one system to the other (staggered approach).

Within the algorithm of numerical integration, the response of the two systems must be determined at the $k$ th time step, for each iteration $j$. The iterative procedure starts from the bridge, with the computation of the RHS of (29). The position of each SMD contact point is de-termined at the beginning of the step according to the rules of the human locomotion described in Section 2 and remains constant during the step. The contact forces $\boldsymbol{f}$ at first iteration are those determined at the end of the previous step, and in subsequent iterations are those at the end of the previous iteration:

$\mathbf{P}^{k, j}=\left(\mathbf{N}^{T} \mathbf{f}\right)^{k, j}=\left(\mathbf{N}^{T}\right)^{k, j=1} \cdot \begin{cases}\mathbf{f}^{k-1} & j=1 \\ \mathbf{f}^{k, j-1} & j>1\end{cases}$

The bridge equations of motion (29) are integrated in incremental form. For $j=1$ the vector of effective loads of the bridge $\Delta \boldsymbol{P}^{* \boldsymbol{k}, \boldsymbol{j}}$ contains the term due to the step initial conditions:

$\Delta \boldsymbol{P}^{* k, j}=\Delta \boldsymbol{P}^{k, j}+\Delta \boldsymbol{P}^{k, i . c}$.

For $j>1$, the increment of nodal loads is computed as:

$\Delta \boldsymbol{P}^{* k, j}=\Delta \boldsymbol{P}^{k, j}=\boldsymbol{P}^{k, j}-\boldsymbol{P}^{k, j-1}$

The bridge solution provides displacements and velocities of contact points, through (14) and (18):

$q_{c b}^{k, j}=N^{k} q_{d c}^{k, j}$

$\dot{q}_{c b}^{k, j}=N^{k} \dot{q}_{d c}^{k, j}$

From (36) and (37) the forcing term (32) for the SMD due to its base motion is computed:

$f_{h}^{k, j}=-\left(k_{c h, h}^{T} N^{k} q_{d c}^{k, j}+c_{c h, h}^{T} N^{k} \dot{q}_{d c}^{k, j}+k_{c h, h}^{T} N^{k} q_{0 c}\right)$

Eq. (31) is integrated in finite form, and an updated value of the forcing term $\boldsymbol{f}^{\boldsymbol{k} \boldsymbol{j}}$ for the bridge is computed as:

$$
\begin{aligned}
f^{k, j}= & -\left(k_{c h, c h} N^{k} q_{d c}^{k j}+k_{c h, h} q_{h}^{k}+c_{c h, c h} N^{k} \dot{q}_{d c}^{k j}+c_{c h, h} \dot{q}_{h}^{k, j}\right. \\
& \left.+\left(k_{c h, c h} N^{k}+c_{c h, c h} N^{k}\right) q_{0 c}\right)
\end{aligned}
$$

In the exact solution, the vectors $\boldsymbol{f}^{\boldsymbol{k} \boldsymbol{j}}$ in (33) and (39) would coincide. Due to the staggered solution, the two vectors differ in principle and their difference provides a measure of the error in two subsequent iterations. Convergence is achieved when, for each pedestrian crossing the bridge, the error err is smaller than a prescribed tolerance $\mu$. Since each pedestrian can have one (SSP) or two (DSP) contact points with the bridge, the difference between forces at subsequent iterations is normalized to the number $n_{c p}$ of contact points:

err $=\sum_{i=1}^{n_{c p}} \frac{1}{n_{c p}}\left|f_{i}^{k, j}-f_{i}^{k, j-1}\right| \leqslant \mu$

The measure (40) is independent of the pedestrian model adopted. If the convergence criterion is satisfied, the algorithm moves to step $k+1$. In the opposite case, the sequence (33)-(40) is repeated, up-dating the iteration counter from $j$ to $j+1$.

\section{Case study: The Seriate Footbridge}

The Seriate steel footbridge (Fig. 5a) is located in the Serio River Park near Milan, Italy. The suspended deck, with an arch rise of $1.3 \mathrm{~m}$, is $63.90 \mathrm{~m}$ long and its width varies between $2.5 \mathrm{~m}$ and $5.0 \mathrm{~m}$. A grid of steel stringers and floor beams support the timber deck (Fig. 5b). The floor beams belong to two classes: the main transverse beams, spaced $3.0 \mathrm{~m}$ apart and characterized by a tapered cross-section, and the secondary IPE 120 transverse beams. The stringers include a couple of IPE 330 edge beams and a central girder with a hollow circular section $(\varnothing=$ $298.5 \mathrm{~mm}$ ). A series of X-braces completes the deck and provides stiffness in the horizontal plane. The suspension system supporting the deck (Fig. 5b) is not symmetric about the vertical plane crossing the longitudinal bridge axis. Four steel pylons, arranged to constitute two Ashaped portal frames, are connected at their top to the main sus-pension cables and to the backstays. The two main suspension cables $(\varnothing=60$ $\mathrm{mm}$ ) support the deck through 42 hangers $(\varnothing=16 \mathrm{~mm})$. Four backstays $(\emptyset=60 \mathrm{~mm})$ link the pylons to the ground. Two lateral stabilizing cables $(\varnothing=40 \mathrm{~mm}$ ) have opposing curvature. During con-struction, all the cables were pre-tensioned.

As a part of the proof tests carried out in July 2012, the dynamic behavior of the footbridge was investigated through dynamic tests performed by Gentile [32]. Operational modal testing under ambient excitation was performed. The instrumentation layout is depicted in Fig. 6. Seven accelerometers at each side of the bridge, numbered from 1 to 14, recorded vertical acceleration. Three instruments (15, 16 and 17) recorded horizontal acceleration. Using different input-output techniques $([39,40])$ typical of operational modal analysis (OMA), 14 vibration modes were identified in the frequency range $0-10 \mathrm{~Hz}$. The frequency of the fundamental mode is $1.03 \mathrm{~Hz}$. Five modes are in the frequency range 1.9-3.0 Hz. Subsequently, to assess the level of vibration due to crossing pedestrians, various loading scenarios were defined and simulated on site, with groups having different number of pedestrians, respectively 4, 8, 16 and 32 . For the proof tests only the maximum acceleration recorded in each test and the location where it took place were considered (see [32]). The test with four walking pedestrians will be analyzed in more detail in Section 6.5.

\subsection{FE model of the bridge}

The FE model of the bridge (Fig. 7) was based on the as-built design data extracted from the blueprints and set up within the ANSYS [45] framework. The girders of the steel grid, the pylons and the hangers are modelled with Timoshenko beam elements named "BEAM 188". Cables and braces are modelled with spar elements transmitting only axial force, named "LINK 180". Timber planks and handrails are modelled as lumped masses on steel grid. The boundary conditions of the footbridge model, as the constraint conditions between adjacent elements, are inferred from the technical drawings. A preliminary non-linear static analysis, using the Newton-Raphson method, accounts for the cables geometric stiffness and for the variation of configuration associated to dead loads. Loads are applied incrementally, dead loads first and the fictitious variation of temperature in cables that simulates their pretension, next. Once the model correctly reproduces the deformed geometry and the design value of tension in cables, the tangent stiffness matrix is computed and a modal analysis is performed by linearizing the system about the equilibrium configuration. No model updating was performed. The values of the natural frequencies for the first experimentally identified 10 modes are listed in Table 1 , where $f_{E}$ and $f_{N}$ denote experimental and numerical values, respectively, and $\operatorname{err}_{f}$ is the percentage error between the two. The first row lists the type of mode shape, namely V-F: vertical-flexural; V-T: vertical-torsional and V-TR: vertical-transversal. V-T denotes coupled modes. Fig. 8 depicts the mode shapes in the frequency range 1.5-4 Hz. Modes 2-5 are in the range of walking induced loads, while modes 5-7 can be excited by running activities [28]. Further details on the derivation of the FE model are found in [32]. 


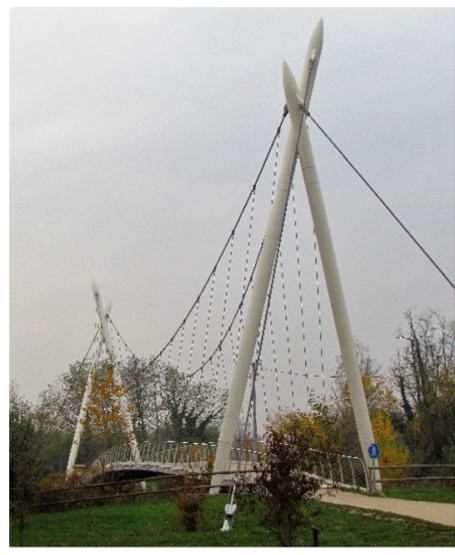

(a)

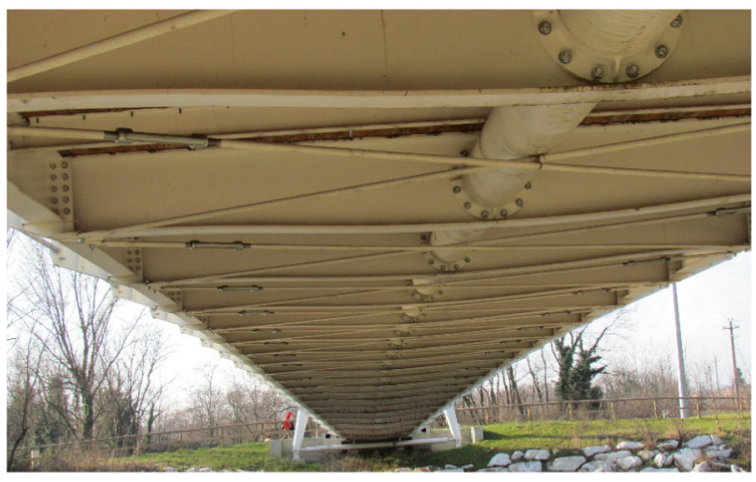

(b)

Fig. 5. Seriate Footbridge: (a) overall view; (b) view of the deck; a stabilizing cable is visible on the left.

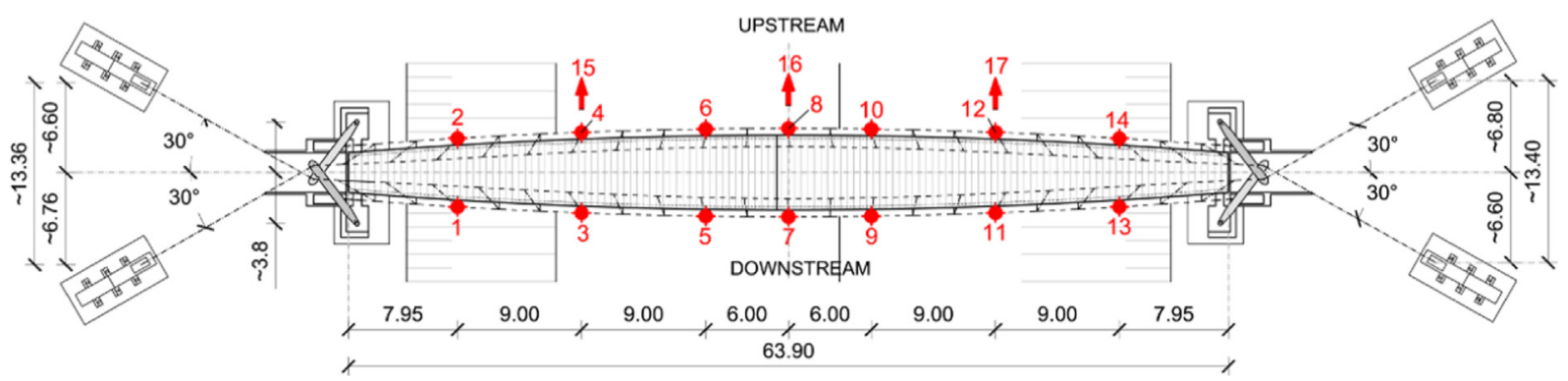

Fig. 6. Experimental campaign, position of accelerometers (after [32]).

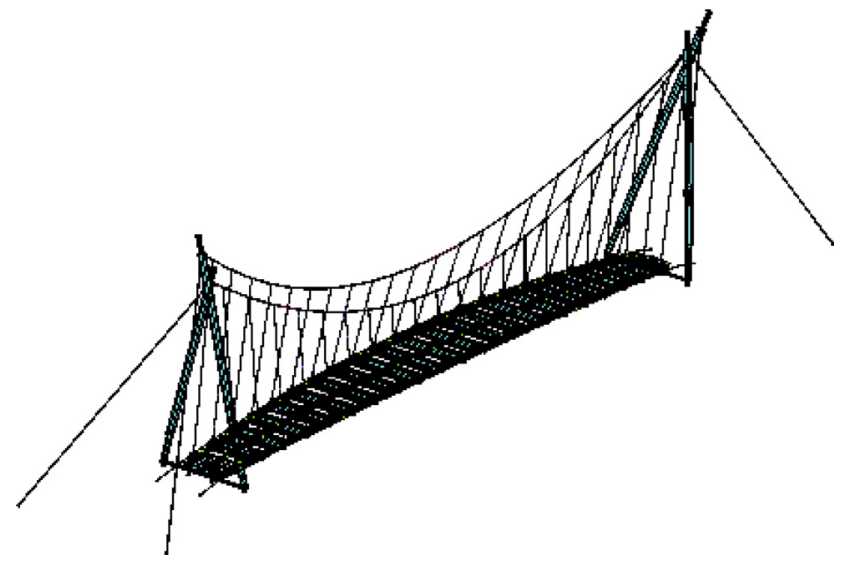

Fig. 7. Finite element model of the Footbridge.

\section{Numerical analysis of dynamic interaction}

The pedestrian model and the iterative procedure for the uncoupled analysis of the pedestrian-footbridge system were implemented in a numerical code in Fortran. The main features of the code are as follows. The shape functions of a shell element relate the vertical displacement of each contact point to three coordinates of the mesh nodes surrounding the contact point (Fig. 4a), namely the vertical displacement and the rotations about the two axes, longitudinal and transversal, in the plane of the bridge deck. The stiffness and mass matrices of the Seriate footbridge model, extracted from ANSYS [45], are input data. The current implementation includes inter-variability. In fact, each pedestrian model has its own properties in terms of mass, stiffness, damping and constant gait characteristics (velocity and step frequency) and can freely walk following any straight trajectory parallel to the bridge axis. Groups of pedestrians can have different spatial configurations, degrees of synchronization, dynamic properties and step frequencies. Full synchronization takes place if the pedestrians walk with the same frequency and in phase, i.e. have the same ${ }^{-} t$ defined in (4) within the gait cycle. Pedestrians start walking at the bridge entrance, since the effect of their initial conditions on the bridge response is negligible. Further details on the code can be found in [41]. In addition, a probabilistic model, that correlates the pedestrians' frequencies with their mutual distances, can be adopted [42].

First, numerical analyses are performed to assess the performance of the iterative procedure and of the numerical code. This aim is pursued through the choice of the numerical integration algorithm, a comparison with a closed-form solution, and an investigation on the convergence of the iterative procedure. Secondly, the bridge response is investigated by considering a few loading scenarios with different

Table 1

Modal properties of the footbridge.

\begin{tabular}{|c|c|c|c|c|c|c|c|c|c|c|}
\hline Mode \# & 1 & 2 & 3 & 4 & 5 & 6 & 7 & 8 & 9 & 10 \\
\hline Type & V-F & V-F & V-T & V-TR & V-F & V-T & V-F & V-T & V-F & V-T \\
\hline$f_{E}[\mathrm{~Hz}]$ & 1.025 & 1.475 & 1.924 & 1.953 & 2.168 & 2.754 & 2.861 & 3.691 & 4.121 & 4.385 \\
\hline$f_{N}[\mathrm{~Hz}]$ & 1.079 & 1.565 & 1.997 & 2.109 & 2.311 & 2.635 & 2.827 & 3.645 & 4.076 & 4.409 \\
\hline$e r r_{f}(\%)$ & 5.22 & 6.07 & 3.77 & 7.99 & 6.58 & -4.33 & -1.17 & -1.24 & -1.10 & 0.55 \\
\hline
\end{tabular}


(a) Mode 2: $f=1.565 \mathrm{~Hz}$

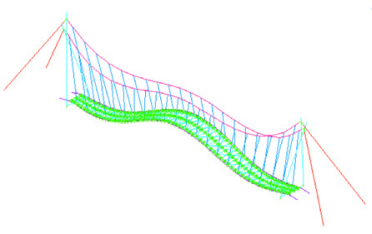

(e) Mode 6: $f=2.635 \mathrm{~Hz}$

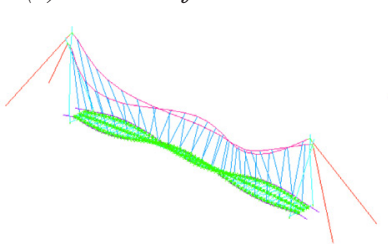

(b) Mode 3: $f=1.997 \mathrm{~Hz}$

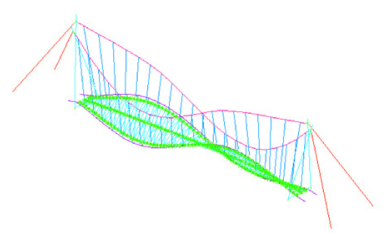

(f) Mode 7: $f=2.827 \mathrm{~Hz}$

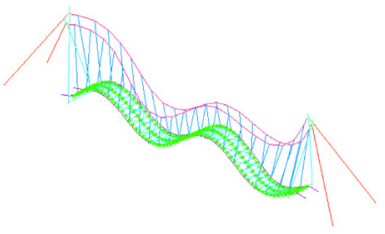

(c) Mode 4: $f=2.109 \mathrm{~Hz}$

(d) Mode 5: $f=2.311 \mathrm{~Hz}$

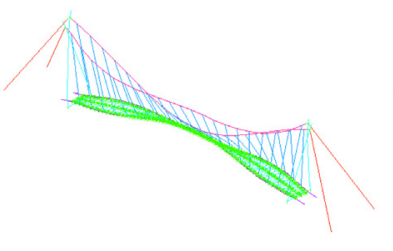

(g) Mode 8: $f=3.645 \mathrm{~Hz}$

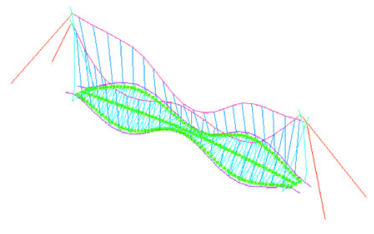

(h) Mode 9: $f=4.076 \mathrm{~Hz}$
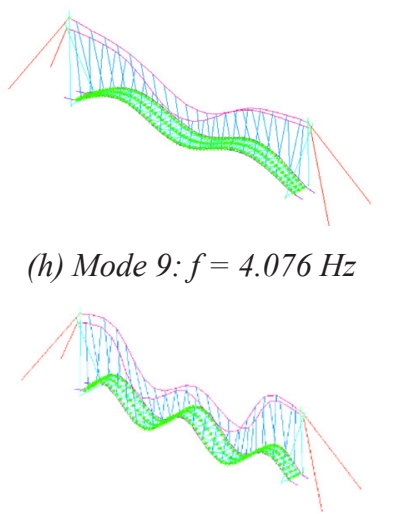

Fig. 8. Seriate Footbridge: numerical mode shapes from $2^{\text {nd }}$ to $9^{\text {th }}$.

spatial configurations. Finally, the comparison with an experimental test, though affected by an incomplete knowledge about pedestrian data, allows for both a discussion on the modelling framework and an evaluation of the bridge response. The time step $\Delta t$ of the numerical integration is equal to $0.005 \mathrm{~s}$, much smaller than $1 / 10$ of the period of the 14th identified mode, and sufficient to discretize accurately the forcing term. The Rayleigh damping matrix is computed for a $1 \%$ damping, a value experimentally identified, on the first and fourth mode. The maximum number of iterations per step is 10 . Unless otherwise specified, the mechanical properties of the pedestrian model are $80 \mathrm{~kg}, 1.80 \mathrm{~m}$ and $1.3 \mathrm{~m} / \mathrm{s}$ (the mean value of a normal distribution in [37]) for mass, height and velocity respectively. Eqs. (6) and (7) provide the leg parameters.

\subsection{Numerical integration of the uncoupled equations of motion}

At a preliminary stage, the equations of motion for the bridge and pedestrian (Eqs. (29) and (31) respectively), were numerically integrated using the Newmark's method. However, the bridge accelerations exhibited spurious high frequency oscillations at time instants when the pedestrian model first applies (or removes) a step contact force. The numerical problem is worsened by the modelling assumption of pointwise contact. For this reason, the bridge equations (29) are integrated using the Hilber, Hughes and Taylor (HHT) algorithm [43]. The contribution of low period modes is damped out without modifications on the lower frequency components. The difference between the two responses in terms of acceleration at mid-span is shown in Fig. 9

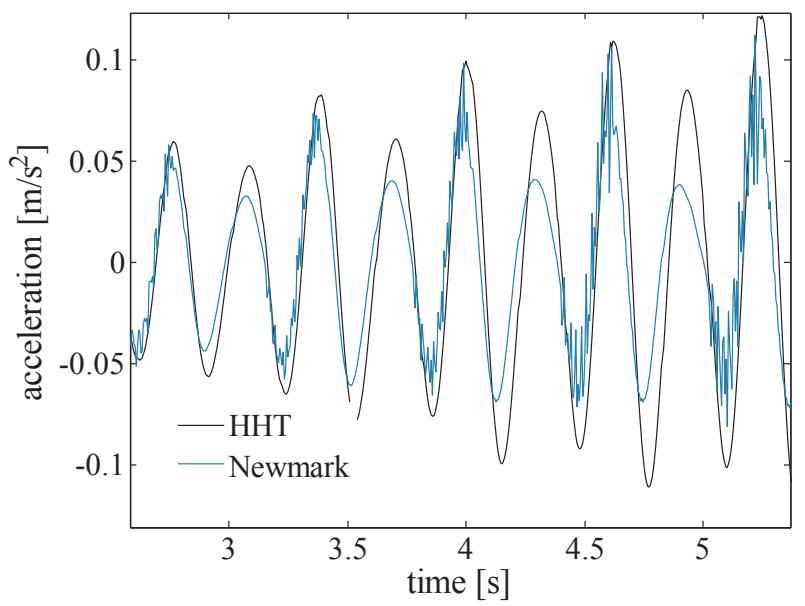

Fig. 9. Newmark vs. HHT method. Time-history of mid-span vertical acceleration, one pedestrian along the axis, $f_{s}=1.6 \mathrm{~Hz}$. for the case of a single pedestrian $\left(f_{s}=1.6 \mathrm{~Hz}\right)$ walking along the bridge axis.

\subsection{Numerical validation of the code}

A numerical validation of the code and of the interaction algorithm is performed preliminarily, by using as benchmark a closed-form solu-tion (Fryba [44]) for the case of a force, equal to the pedestrian's weight, travelling at constant speed on a simply supported beam of uniform cross-section. The case study is an ideal undamped 1D simple supported beam, $48 \mathrm{~m}$ long, with a rectangular cross-section $(3.0 \mathrm{~m}$ wide and 0.73 $\mathrm{m}$ deep), discretized in 48 elements. Young's modulus is $210 \mathrm{GPa}$, density is $2.5 \times 10^{5} \mathrm{~kg} / \mathrm{m}^{3}$. The beam natural frequencies are listed in Table 2, where $\mathrm{V}$ and $\mathrm{T}$ denote vertical and transversal modes, respectively. Vertical modes are either symmetric (s) or anti-symmetric (as) about mid-span. Three models of increasing accuracy simulate numerically the travelling pedestrian $\left(\mathrm{W}=700 \mathrm{~N}, \mathrm{f}_{\mathrm{s}}=1.6 \mathrm{~Hz}\right)$ as follows. The first model is a constant static force; the second is a travelling couple of single foot forces reproducing the human gait in Fig. 1a, with magnitude varying in time according to Eq. (1), same weight of the static force; and the third is a SMD model, with the same gait. For a positive validation of the code, the solution to the static force should match the closed-form solution. Discrepancies between the two more accurate modelling of the pedestrian and between these and the static case must be consistent with the dynamic nature of these loads.

The comparison in terms of mid-span vertical displacement is presented in Fig. 10a. The numerical solution due to the static force exactly matches the analytical one. As expected, the displacements due to the travelling single foot forces oscillate about the closed-form solution, with the same frequency of the steps. The SMD model induces displacements having a similar pattern but a lower amplitude of oscillations. The result can be justified by the damping added by the model to the overall system. In the last two cases, the bridge response shows a time-delay attributed to the difference between the human gait and the uniform motion adopted for the static force. Fig. 10b shows the midspan vertical acceleration. The match between the closed form solution and the travelling static force is very good. Greater accelerations are detected for the case of the dynamic force and, to a lesser extent, of the SMD model, consistently with the results in terms of displacements. Taking into account the values of the natural frequencies, the Fourier spectra of the accelerations (Fig. 10c and d) deliver a clear picture of the dynamic response. In the static case, the beam response at mid-span in Fig. 10c is dominated by the frequencies of the vertical symmetric modes. In the dynamic case in Fig. 10d the beam is responding also to the step frequency and its multiples. The difference between the first natural frequency of the beam $(0.195 \mathrm{~Hz})$ and the step frequency $(1.6$ $\mathrm{Hz}$ ) justifies the match in terms of displacements and the 
Table 2

Modal properties of the simply supported beam. V/T vertical/transverse mode; s/as symmetric/antisymmetric.

\begin{tabular}{|c|c|c|c|c|c|c|c|c|c|c|c|}
\hline Mode \# & 1 & 2 & 3 & 4 & 5 & 6 & 7 & 8 & 9 & 10 & 11 \\
\hline Type & V-s & V-as & $\mathrm{T}$ & V-s & $\mathrm{V}$-as & $\mathrm{T}$ & V-s & V-as & $\mathrm{T}$ & V-s & V-s \\
\hline$f[\mathrm{~Hz}]$ & 0.195 & 0.779 & 0.800 & 1.752 & 3.113 & 3.183 & 4.860 & 6.991 & 7.105 & 8.764 & 9.505 \\
\hline
\end{tabular}

discrepancy in terms of accelerations. In Fig. 10d, the peak about $4.8 \mathrm{~Hz}$ is justified by the match between the $7^{\text {th }}$ natural frequency and the $2^{\text {nd }}$ harmonic of the periodic load. The same peak is found in Fig. 10c also. The results show that the analytical formulation and the computer code implementing it simulate correctly the dynamic behavior of the beam.

\subsection{Evaluation of the performance of the iterative procedure}

The iterative procedure is a key point in the solution of the uncoupled equation of motion. To evaluate its properties of convergence and precision three loading scenarios centered along the bridge axis are examined. These are: (1) a single pedestrian; (2) six pedestrians walking in a longitudinal row, spaced $2 \mathrm{~m}$ apart; and (3) nine pedestrians uniformly distributed on three longitudinal rows spaced $0.75 \mathrm{~m}$ and $2 \mathrm{~m}$ apart in the transverse and longitudinal direction, respectively. Pedestrians have the same step frequency and weight $(1.6 \mathrm{~Hz}$ and $700 \mathrm{~N})$ but are not synchronized. Each loading scenario is analyzed four times, without iteration and with three decreasing values of the error tolerance $\mu$ in (40): $0.1 \mathrm{~N}, 0.01 \mathrm{~N}$ and $0.001 \mathrm{~N}$. In a fifth analysis, without iteration, the groups of six and nine pedestrians have different

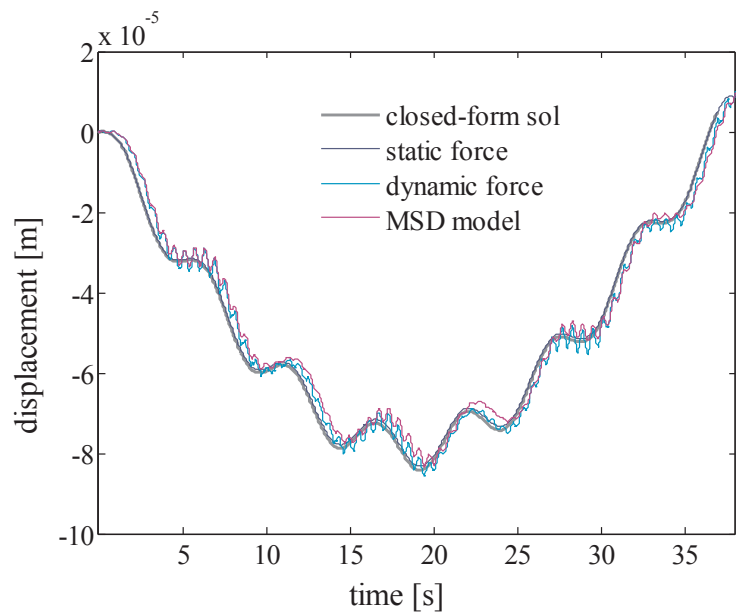

(a)

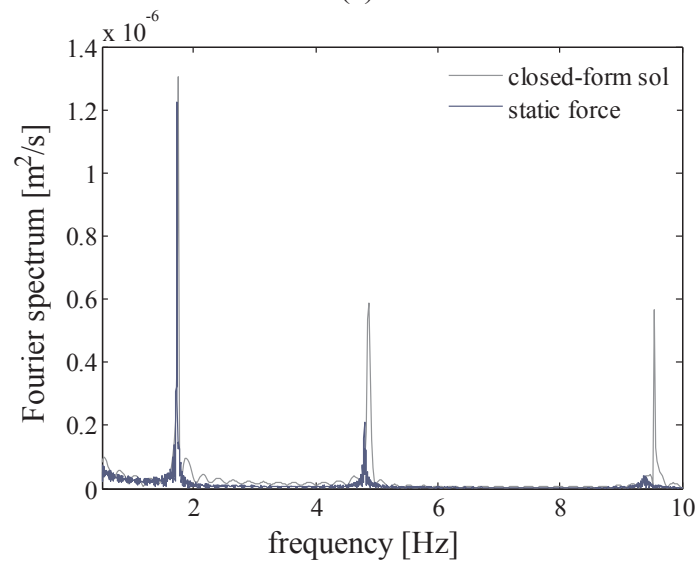

(c) step frequencies, in the range $1.6-2.1 \mathrm{~Hz}$ and $2.1-2.3 \mathrm{~Hz}$ respectively.

The comparison among analyses, for each loading scenario, is performed in terms of acceleration time-history at mid-span central node. The results $\boldsymbol{a}_{0.001}$, corresponding to the smallest tolerance, are practically exact and are assumed as reference values. The percentage difference $\Delta$ between the current $\boldsymbol{a}_{t o l}$ and the reference $\boldsymbol{a}_{0.001}$ acceleration, normalized to the latter, is computed at each at each time step $k$ :

$\Delta_{k}=\left(a_{0.001}-a_{t o l}\right)_{k} \times\left(100 / a_{0.001, k}\right)$

Acceleration values close to zero, producing high but not significant values of $\Delta$, are filtered out from the computation of (41). The precision of the iterative algorithm is measured through the maximum percentage difference $\Delta_{\max }$ and the percentage difference $\Delta a_{\max }$ corresponding to the maximum value of $\boldsymbol{a}_{0.001}$. Table 3 shows, for each loading sce-nario and for each case, the total number $N_{\text {tot }}$ of iterations, the max-imum number $n_{\max , \Delta t}$ of iterations in a single time step and the values of $\Delta_{\max }$ and $\Delta a_{\max }$. The results show that: (a) $\Delta_{\max }$ decreases as the toler-ance, denoting a convergence of the iteration algorithm; (b) the most significant engineering parameter $\Delta a_{\max }$ is much smaller than $\Delta_{\max }$. The number of iterations increases as the number of pedestrians, but not

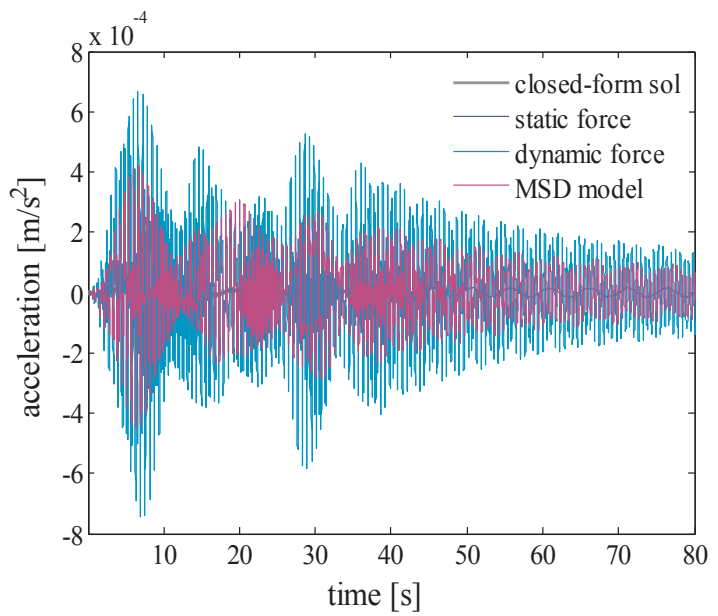

(b)

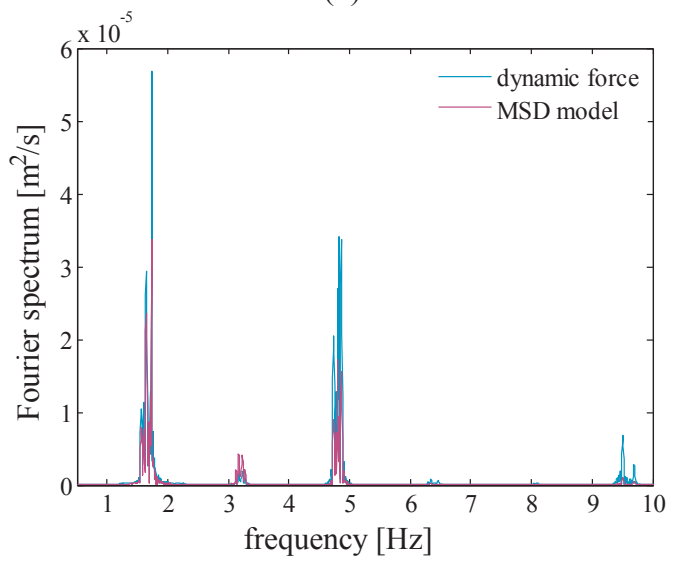

(d)

Fig. 10. 1D bridge, closed-form vs numerical solutions. Time-history of mid-span vertical: (a) displacement; (b) acceleration. Fourier spectrum of acceleration: (c) closed form solution and static force; (d) dynamic force and MSD model. 
Table 3

Performance and convergence properties of the iterative procedure.

\begin{tabular}{|c|c|c|c|c|c|c|c|c|c|c|c|c|}
\hline \multirow[t]{2}{*}{ Tolerance $[\mathrm{N}]$} & \multicolumn{4}{|c|}{1 pedestrian } & \multicolumn{4}{|c|}{6 pedestrians } & \multicolumn{4}{|c|}{9 pedestrians } \\
\hline & $\mathrm{N}_{\text {tot }}$ & $\mathrm{n}_{\text {max }, \Delta \mathrm{t}}$ & $\Delta_{\max }[\%]$ & $\Delta \mathrm{a}_{\max }[\%]$ & $\mathrm{N}_{\text {tot }}$ & $\mathrm{n}_{\max , \Delta \mathrm{t}}$ & $\Delta_{\max }[\%]$ & $\Delta \mathrm{a}_{\max }[\%]$ & $\mathrm{N}_{\text {tot }}$ & $\mathrm{n}_{\max , \Delta \mathrm{t}}$ & $\Delta_{\max }[\%]$ & $\Delta \mathrm{a}_{\max }[\%]$ \\
\hline 0.1 & 11,789 & 1 & 0.03 & 0.0015 & 11,831 & 2 & 0.07 & 0.003 & 11,813 & 1 & 0.6 & -0.0162 \\
\hline 0.01 & 12,890 & 2 & 0.02 & 0.0002 & 11,831 & 2 & 0.06 & 0.0011 & 20,008 & 2 & 0.13 & 0.0072 \\
\hline 0.001 & 20,675 & 2 & / & / & 21,860 & 2 & / & / & 22,092 & 2 & / & / \\
\hline No iteration same $f_{s}$ & / & / & -7.3 & -1.8 & l & / & -24.5 & -5.6 & / & / & -28.1 & -13.8 \\
\hline No iteration different $f_{s}$ & I & / & / & / & / & I & -20.4 & -1.97 & / & / & -27 & -0.5 \\
\hline
\end{tabular}

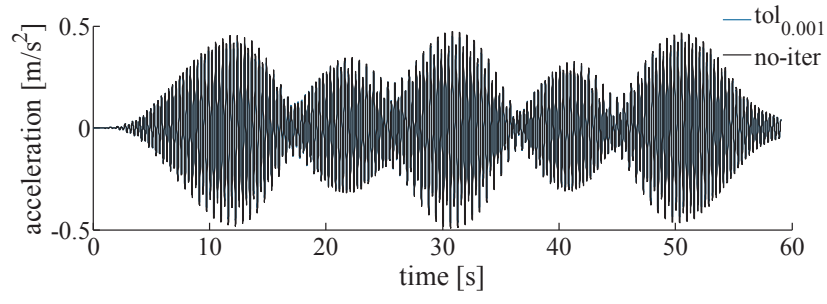

Fig. 11. Six pedestrians walking in a row: time-history of mid-span vertical acceleration with and w/o iteration.

with a monotonic trend. With iterations, a tolerance equal to $0.1 \mathrm{~N}$ suffices to provide accurate results. Without iterations, both $\Delta_{\max }$ and $\Delta a_{\max }$ are reduced when each pedestrian walks with his/her own step frequency. In this case, the results without iterations, with $\Delta a_{\max }$ smaller than $2 \%$, provide a good estimate of the bridge response.

The time-history of mid-span acceleration is depicted in Fig. 11 for the cases of six pedestrians, with tolerance equal to $0.001 \mathrm{~N}$ and without iteration. The general pattern of the response is the same, making it difficult to distinguish the two curves, but the purely stag-gered solution overestimates the response. This result is shown clearly in Fig. 12a, a zoom in the time interval 10-11.5 s. Moreover, Fig. 11 shows a beat phenomenon due to the frequency range excited by the pedestrianinduced load, as confirmed by the Fourier spectrum in Fig. 12b.

\subsection{Groups of pedestrians with different trajectories}

The bridge response is analyzed for three trajectories of the same group of six people in a longitudinal row, spaced $2 \mathrm{~m}$ apart, to investigate the importance of the 3D modelling. Pedestrians walk either along the bridge axis or along two parallel lines, with a left or right eccentricity of $1 \mathrm{~m}$ from the axis. The six SMD pedestrians are uncorrelated and unrestricted. They have a common weight $(700 \mathrm{~N})$ and height $(1.70 \mathrm{~m})$, but different step frequencies $(1.843 \mathrm{~Hz} ; 2.057 \mathrm{~Hz}$; $1.675 \mathrm{~Hz} ; 1.776 \mathrm{~Hz} ; 1.634 \mathrm{~Hz} ; 1.799 \mathrm{~Hz}$ ) drawn from a normal distribution. Each pedestrian crosses the bridge in about $49 \mathrm{~s}$ and the last pedestrian exits the bridge about $8 \mathrm{~s}$ after the first.

The relevant response parameters are the time histories of vertical displacements and accelerations, evaluated in seven transverse sections of the deck at three points. Two are at the handrails base and the third on the deck longitudinal axis (Fig. 13). Fig. 14 a/b/c show, respectively, the time-histories of displacements at nodes 16, 17 and 18 (at section \#6, 3/4 of the span), for the three cases under study. The torsional effect induced by the eccentric transits is larger when the pedestrians cross the section under study and in the nodes closer to the pedestrians' trajectory, while vanishing for the central trajectory (Fig. 14b).

Fig. 15 shows the peak values of acceleration, at the sections and nodes in Fig. 13. Thick lines bound the seven sections. For eccentric trajectories, extreme values are on the same side of the eccentricity and show a relevant difference with values on the opposite side of the same section. The torsional effect produced by the central trajectory is much smaller but not zero, vanishing only for node 11 at mid-span where the three peaks are practically coincident.

Fig. 16 shows a few acceleration time histories, at nodes 4 (section \#2, left, right and central trajectory), 11 (section \#4, central trajectory) and 18 (section \#6, left and right trajectory). Maximum accelerations are due to the eccentric configurations and are detected at lateral nodes 4 and 18 when the pedestrians cross the sections \#2 and \#6 (Fig. 16a and $f$ ). The couples of nodes symmetrically placed about the mid-span but on the opposite sides of the bridge exhibit a specular behavior under eccentricity having opposite signs (Fig. 16a, f and c, d). A central trajectory produces similar time histories in the three sections; the results for Sections 2 and 4 are shown in Fig. 16b and d. The trajectory does not affect the response only at mid-span (e.g. Fig. 16e, node 11). Fig. 17 depicts the Fourier spectra of the time histories in Fig. 16. Since the six pedestrians walk with different frequencies a broad frequency content is found, containing the first and second harmonic of the load and the bridge natural frequencies. Spectra due to eccentric transit have

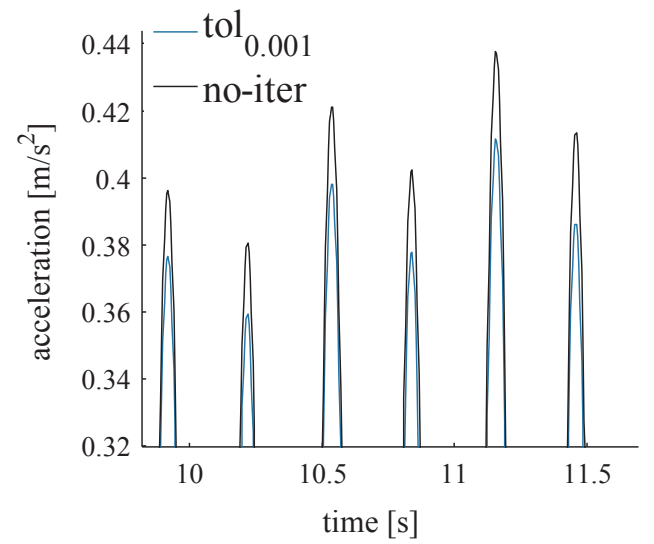

(a)

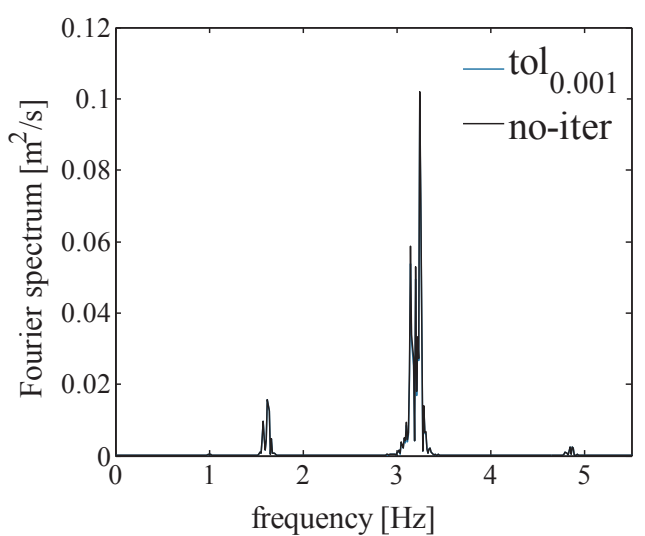

(b)

Fig. 12. Six pedestrians walking in a row, mid-span vertical acceleration with and w/o iteration: (a) zoom in the interval 10-11.5 s; (b) Fourier spectrum. 


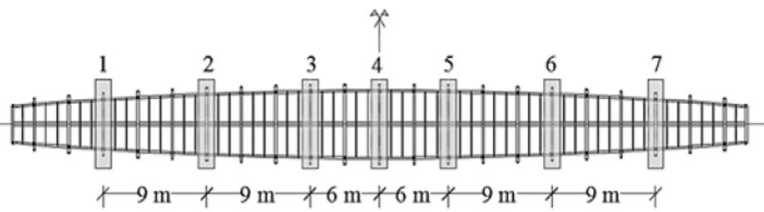

(a)

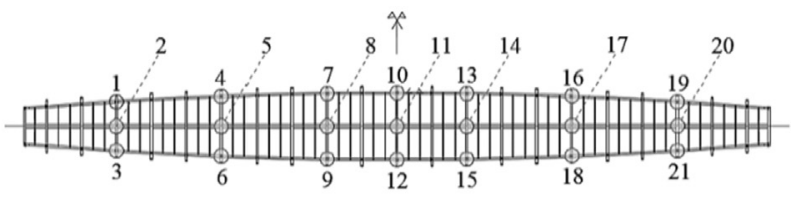

(b)

Fig. 13. Evaluation of the bridge response: (a) cross sections; (b) control nodes for vertical acceleration.

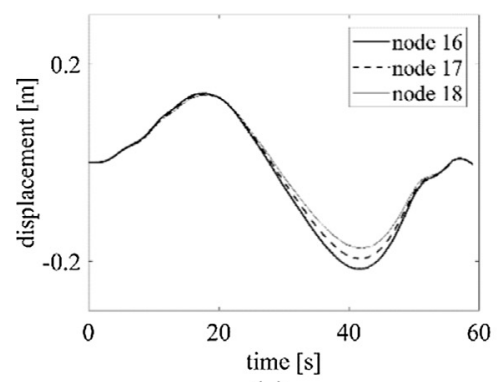

(a)

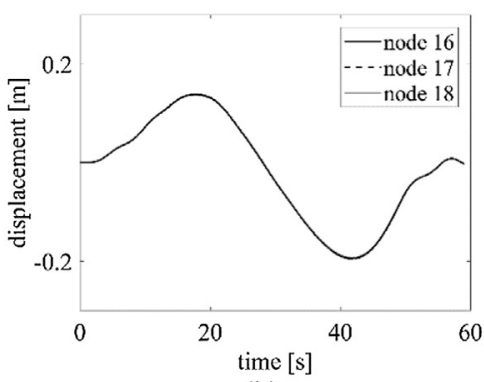

(b)

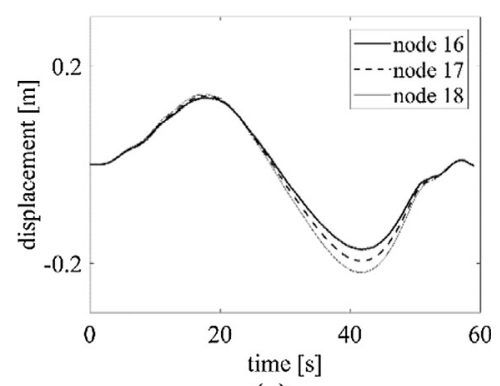

(c)

Fig. 14. Six pedestrians in a row. Displacement time-history of nodes at section 6 for trajectories having: (a) left; (b) no and (c) right eccentricity.

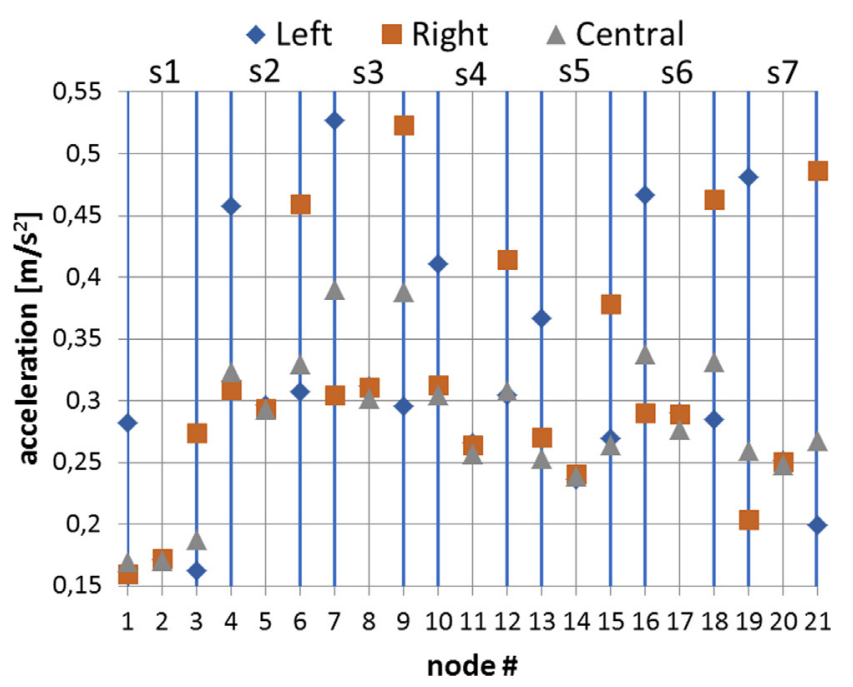

Fig. 15. Six pedestrians in a row. Peak values of vertical acceleration due to a left, right and central trajectory.

a common pattern (Fig. 17a, c and d, f), different from that due to a centered transit (Fig. 17b and e). The response spectra are unaffected by the trajectory only at mid-span (e.g. Fig. 17e, node 11). The modal shapes of the $3^{\text {rd }}$ mode (Fig. $8 \mathrm{~b}, \mathrm{f}_{3}=1.997 \mathrm{~Hz}$ ) and of the $8^{\text {th }}$ mode (Fig. $8 \mathrm{~g}, \mathrm{f}_{8}=3.645 \mathrm{~Hz}$ ), two torsional modes with a node around mid-span close to the bridge axis [32], justify the outcome of the analyses. Results show that the bridge response is correctly described by the proposed numerical approach and highlight the significant effect in-duced by different trajectories.

\subsection{Comparison with experimental results}

As stated in Section 4, dynamic testing of the footbridge under walking pedestrians was performed as a part of the proof tests. The aim of the tests was only to assess the level of vibration under loading scenarios with different numbers of pedestrians and data on pedestrians were not collected. Hence, weight, height, step frequency, velocity, mutual positions, trajectories, entry side on the bridge are unknown. In spite of the lack of data, a comparison between experimental and numerical results is performed, extrapolating some information from the recorded signals, and adopting reasonable estimates for the remaining parameters. Such a comparison aims to evaluate the proposed framework of analysis and the effects of inter- and intra-variability modeling.

The parameters were chosen as follows. For the experimental test with four walking pedestrians analyzed here, it was estimated by Gentile (who performed the tests, see [32]) that the pedestrians were in a rectangular configuration, spaced $80 \mathrm{~cm}$ and $40 \mathrm{~cm}$ apart in the longitudinal and transverse direction, respectively. Reasonable choices for weight and height of Italian male pedestrians are $750 \mathrm{~N}$ and $1.75 \mathrm{~m}$. It was assumed that pedestrians entered the bridge from the right side in Fig. 6. In light of the results in Section 6.4, a centered trajectory is a neutral choice. The pedestrians' dominant velocity and frequency are roughly identified from the time histories and Fourier spectra of acceleration. Based on the tests duration (see Fig. 18), the velocity $v_{h}$ is assumed equal to $1.7 \mathrm{~m} / \mathrm{s}$. The Fourier spectra of the signals (in Fig. 19) denote a peak at about $2 \mathrm{~Hz}$. This frequency is assumed as a tentative value for $f_{h}$. Experimental and numerical results are compared in terms of vertical acceleration and related Fourier spectra at selected positions of accelerometers (see Fig. 6).

In a first analysis, inter-variability is neglected and the pedestrians, walking at $f_{h}=2 \mathrm{~Hz}$, are fully synchronized. Six positions of accelerometers are considered, two at mid-span $(7,8)$, two in the first halfspan $(1,4)$ and two in the second half-span $(11,14)$. Numerical accelerations (Fig. 18) at the opposite sides of each sections are similar, since the trajectory is centered. A significant beating phenomenon is visible in the numerical response, more pronounced than in the experimental response. In the numerical results, the discrepancy between the responses at positions 1,4 and 11, 14 is larger than that in the experimental response. The closer match in terms of general pattern is found at mid-span, where torsional modes have a node and the effect of trajectory eccentricity vanishes, even though the numerical response underestimates the peak values. The Fourier spectra (Fig. 19) show that the frequency content of the numerical response is concentrated around the step frequency and its multiples, with a dominant peak at about 2 $\mathrm{Hz}$, whose magnitude is larger than in the experimental response. On the other hand, this shows a much wider frequency content, with components about 3 and $6 \mathrm{~Hz}$ also. When the numerical results in Figs. 19 and 17 are compared, it appears clearly that the pedestrians' configuration, the trajectory eccentricity and the inter-variability in terms of step frequency have a dominant effect on the response.

The results in Fig. 19 suggest releasing the unrealistic assumption of full synchronization. Thus, a second and third case are considered, 
(a) Left, node \#4

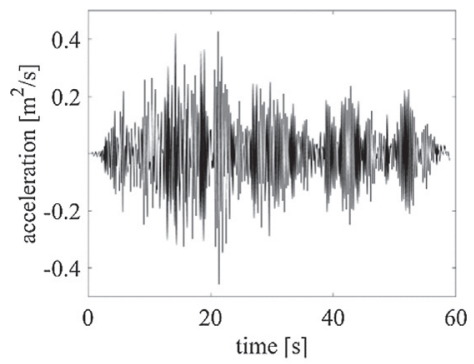

(d) Left, node \#18

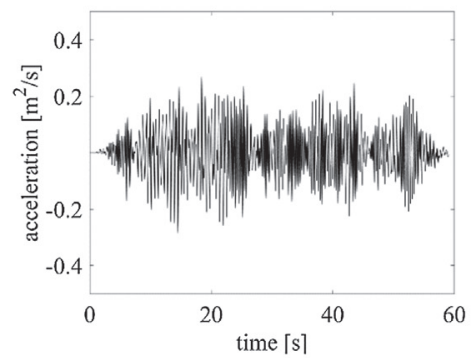

(b) Central, node \#4

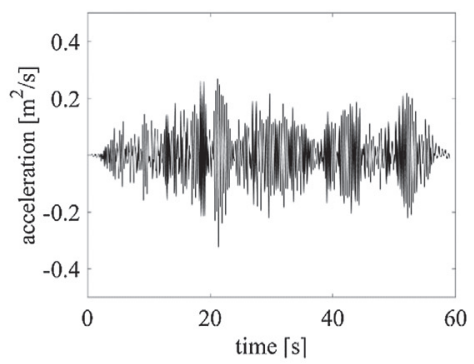

(e) Central, node \#11

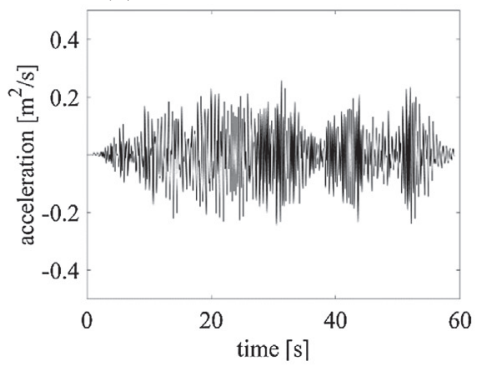

(c) Right, node \#4

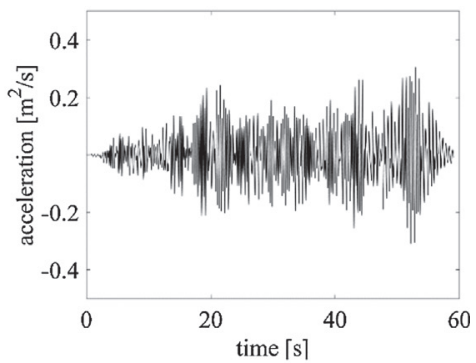

(f) Right, node 18

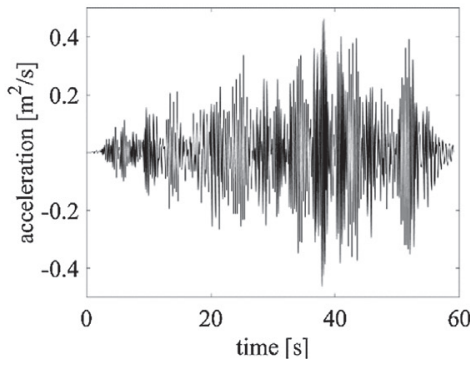

Fig. 16. Six pedestrians in a row. Time-history of vertical acceleration at nodes 4,11 and 18 for left, central and right trajectories.

where pedestrians are in a row-synchronization and in phase, with $f_{h}=$ $2.05 \mathrm{~Hz}$ and $1.95 \mathrm{~Hz}$ in the first and second row respectively. In the third case, a delay of $0.1 \mathrm{~s}$ (about $20 \%$ of $T_{e}$ ) in the gait cycle is imposed between two subsequent pedestrians. The phase lag is constant during the bridge crossing. Fig. 20 presents the numerical results at positions 1 , 4 and 8. The comparison with the results in Figs. 18 and 19 shows clearly that a slight modification of the gait parameters is able to modify the response significantly. The best match for accelerations is found for the case of row-synchronization while the introduction of a phase difference produces a general underestimation of the signal. The Fourier spectra are intermediate between the numerical and the ex-perimental ones in Fig. 19. The sharp peak at about $2 \mathrm{~Hz}$ of the fully synchronized case becomes wider but not as the experimental one. The experimental high frequency components at about $6 \mathrm{~Hz}$ are absent in the numerical response, while those at about $3 \mathrm{~Hz}$ are visible in position 8 , when a phase difference is accounted for. Finally, Fig. 21 summarizes the results in terms of peak values. The results point out a significant torsion effect in the experimental results and a large scatter among numerical results for different synchronizations. However, experimental and numerical extreme values above $1.2 \mathrm{~m} / \mathrm{s}^{2}$ produce the same negative outcome in terms of bridge serviceability assessment.

\section{Conclusions}

This work presents the derivation of an analytical formulation for the footbridge-walking pedestrian coupled problem in the vertical (a) Left, node \#4

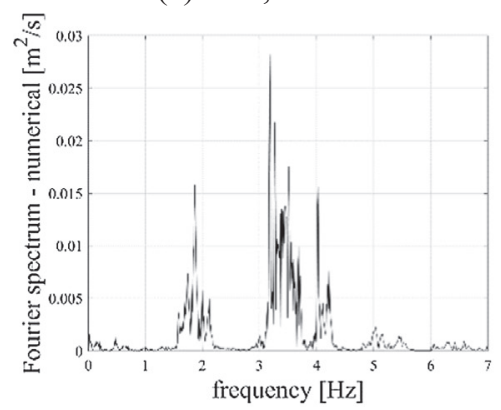

(d) Left, node \#18

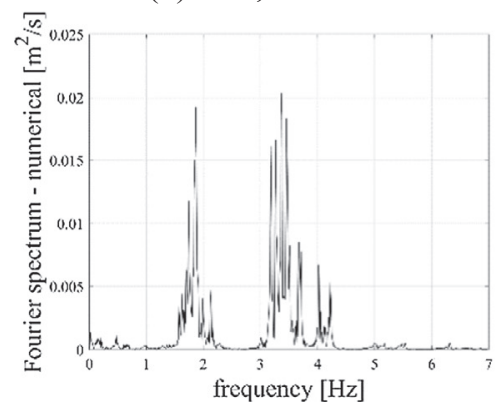

(b) Central, node \#4

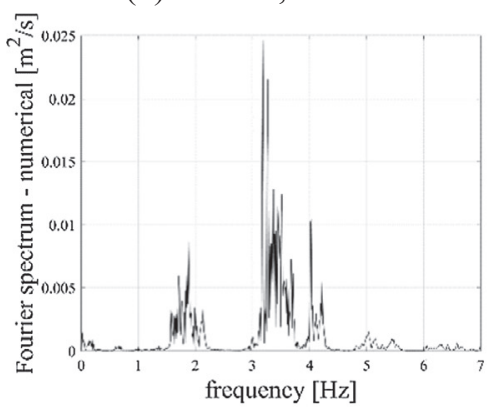

(e) Central, node \#11

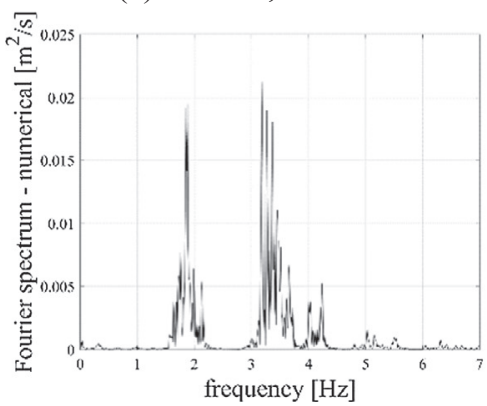

(c) Right, node \#4

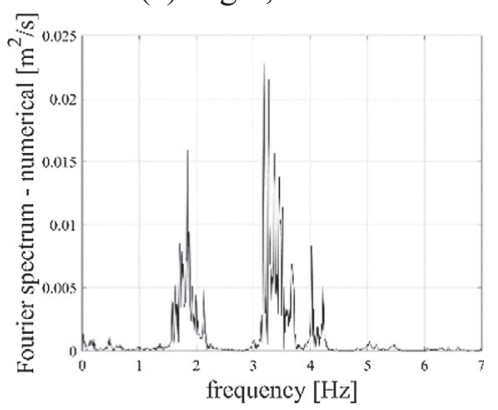

(f) Right, node \#18

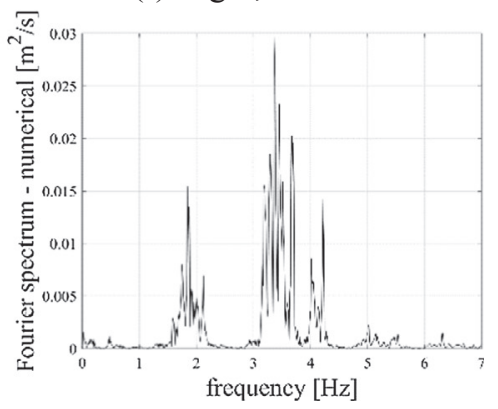

Fig. 17. Six pedestrians in a row. Fourier spectrum of vertical acceleration at nodes 4,11 and 18 for left, central and right trajectories. 

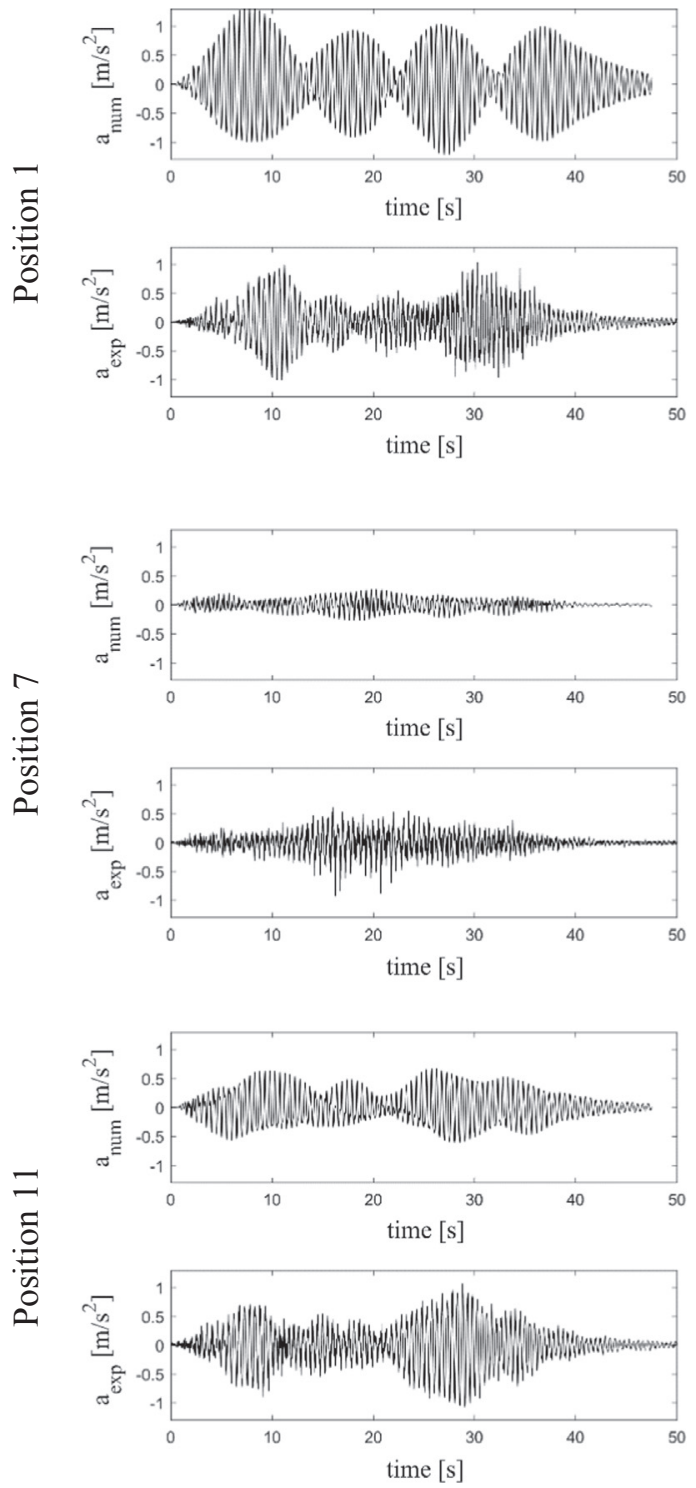
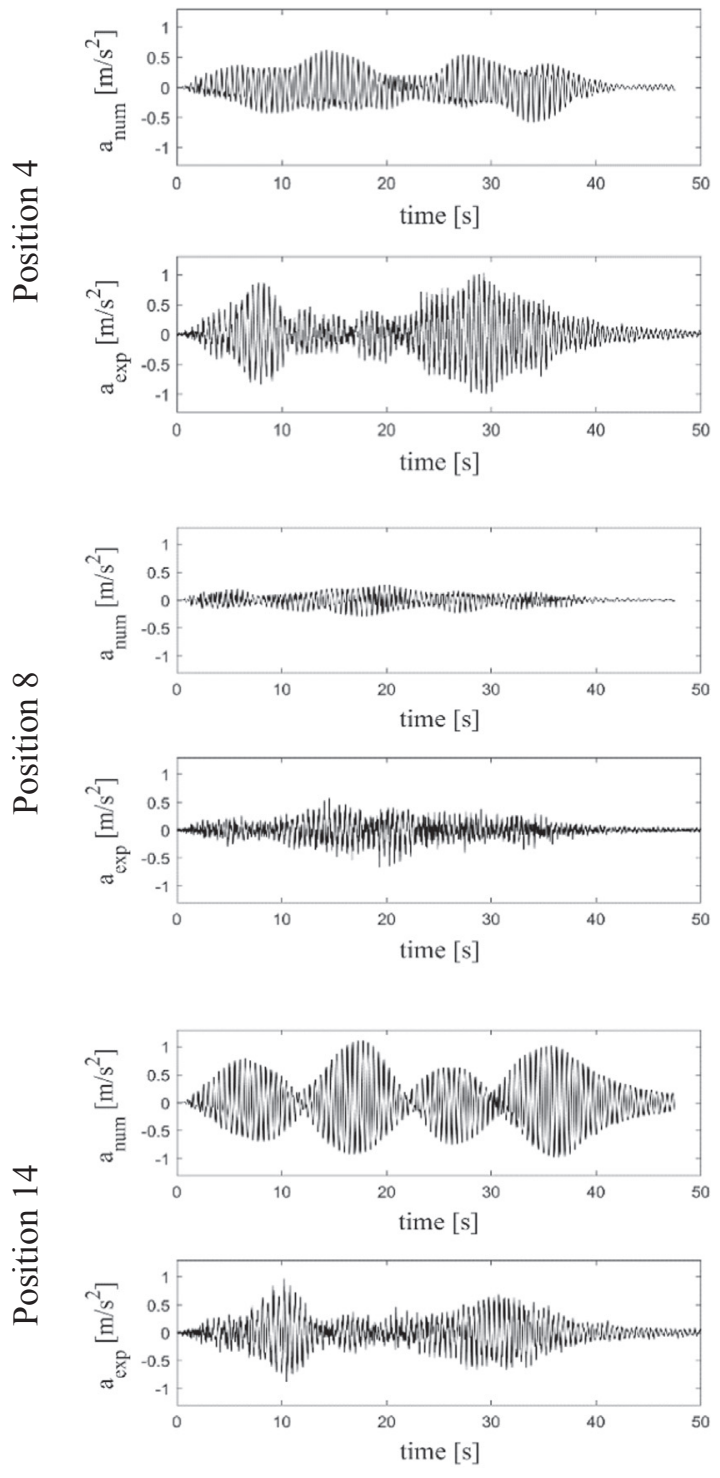

Fig. 18. Four pedestrians, numerical $\left(a_{\text {num }}\right)$ vs experimental $\left(a_{\text {exp }}\right)$ time-history of vertical acceleration at selected positions.

direction. The derivation of the coupled equation of motion is based on the Lagrange's equation and relies on a discrete modelling framework. The bridge is described through a standard 3D FE model, in principle the same adopted in design. A new bipedal SMD model represents the pedestrian. The model matches reality in simulating the sequence of single and double support phases of human gait and transmitting vertical contact forces only through two compliant and damped legs. An equivalent "biomechanical" force provides energy for the vertical oscillations. Perfect contact takes place at massless contact points. The proposed solution strategy, based on the forced uncoupling of the equations associated with an iterative procedure, allows for the separate integration of the two systems. In the uncoupled solution, the footbridge is subjected to the contact forces transmitted by the pedestrians, and the pedestrians are subjected to the bridge motion at their contact points (feet). This result could have been reached by inspection, but the formally complete approach clarifies the properties and the advantages of the solution. First, there is no direct mechanical coupling among pedestrians: respecting the inter-subject variability, each pedestrian can have his own properties and horizontal motion. Second, social effects modifying the gait of pedestrians can be analyzed outside the coupled framework: in fact, only the position of pedestrians and their contact forces affect the coupled solution. Social coupling does not imply mechanical coupling: the pedestrians' trajectory and a variable rate of progression can be determined in a separate analysis and provided as input data. Third, the motion perceived at any instant by each pedestrian can be determined, to assess his degree of comfort along the bridge.

The analytical formulation is implemented in a research code previously developed to analyze the VBI problem. The case study is a lively suspension footbridge [32]. The adoption of the standard 3D FE model, developed in ANSYS [45], overcomes the limits of reduced-order modelling, often restricted to the first mode, and allows for a pedestrians' 2D trajectory. From the point of view of implementation, the main advantage of the new bipedal model is preserving linearity even though the legs parameters vary during the double support phase. Disregarding intra-variability is its most limiting assumption, even though advantageous in terms of ease of implementation and being well documented in the literature. However, intra-variability is consistent with the proposed analytical framework and can be easily introduced, since horizontal and vertical motion of the SMD model are uncoupled, and the former is assumed to be known. For the same reason, the straight trajectory parallel to the bridge axis implemented in this study can be substituted by curved or zigzag trajectories.

Numerical analyses in the linear range, performed preliminary to 

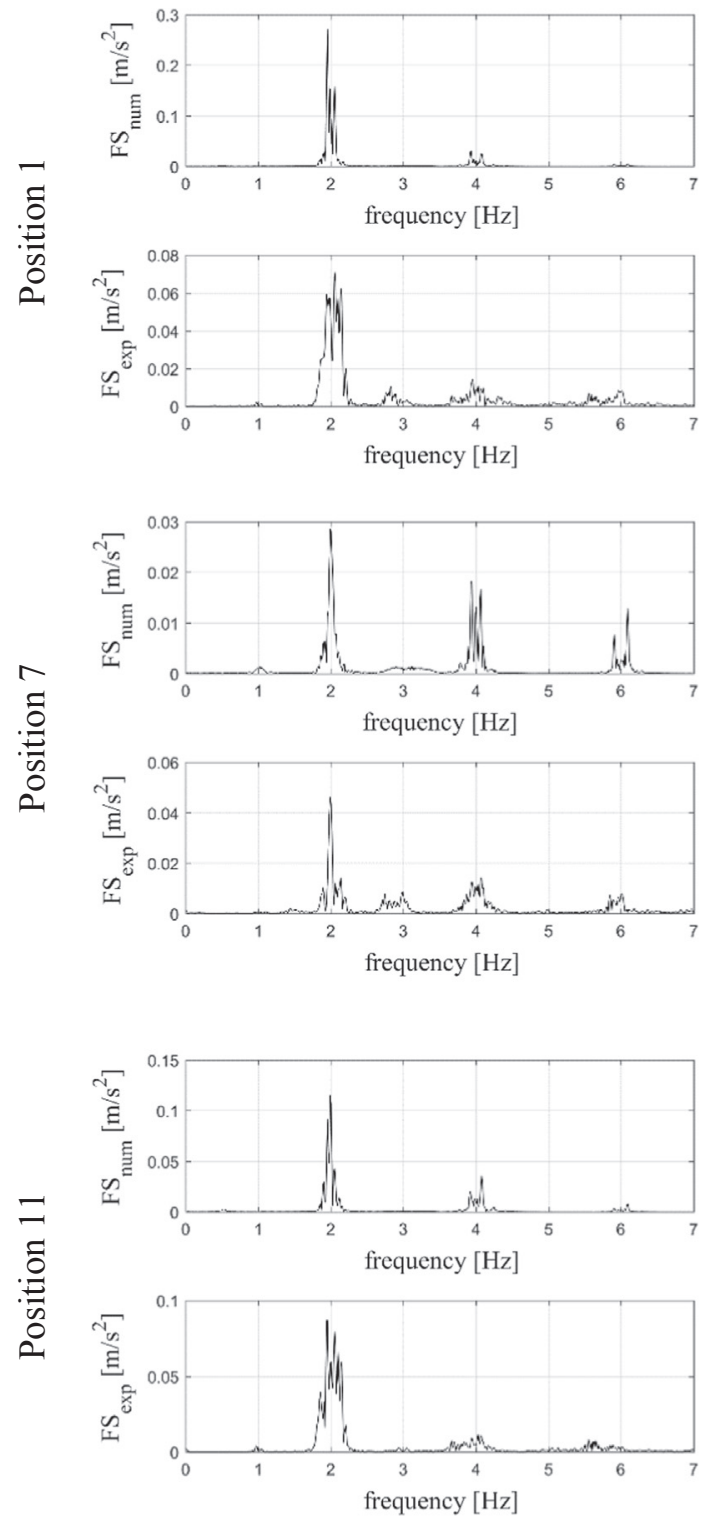

Fig. 19. Four pedestrians, numerical $\left(\mathrm{FS}_{\text {num }}\right)$ vs experimental $\left(\mathrm{FS}_{\text {exp }}\right)$ Fourier Spectrum of vertical acceleration at selected positions.

investigate the problems related to the instantaneous application/removal of forces on the bridge, lead to the adoption of the well-known HHT algorithm to eliminate a spurious high frequency content. Numerical tests confirmed the positive performance of the iterative procedure. A numerical validation of the code is obtained by reproducing a closed form solution for a static load moving continuously on a simply supported beam. The walking SMD model, and its counterpart in terms of GRFs simulating the human gait (dynamic loads), showed a match with the closed form solution in terms of displacement and discrepancies in terms of accelerations. These are consistent with the dynamic properties of both the crossed beam and the applied load. In particular, the damping effect induced by the SMD model on the response is detected clearly.

A further set of analyses concerned groups of pedestrians with different spatial configurations, to evaluate, also by comparison with experimental results, the effect of inter-variability and spatial configuration of pedestrians. The numerical results for six pedestrians in a row depend on the eccentricity of the trajectory and highlight the importance of retaining the 3D bridge modeling. An experimental test with four pedestrians in a rectangular configuration was simulated, even though most of the test arrangement was not known. A reasonable
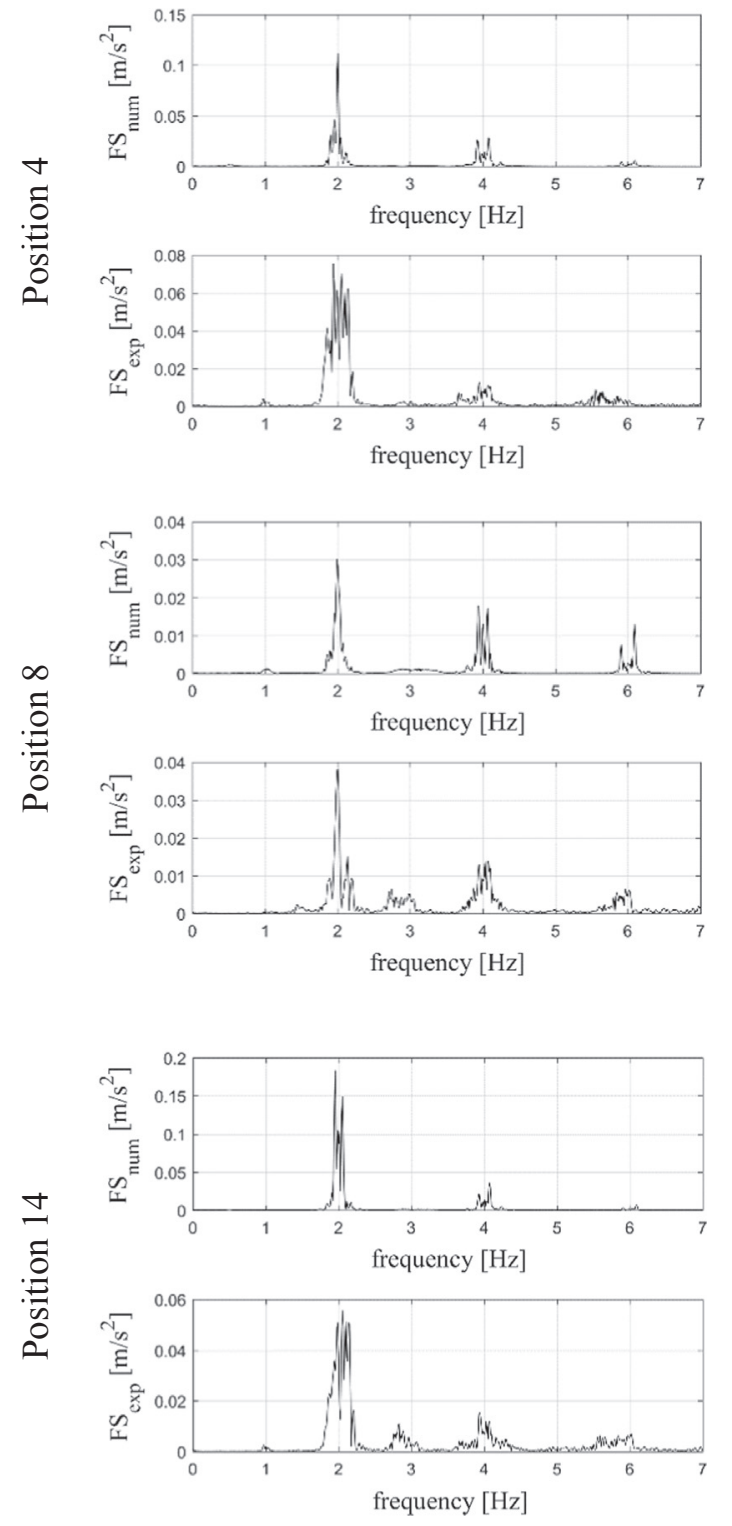

set of parameters was chosen, and three different degrees of intervariability were assumed. None of the cases analyzed matched well the experimental results, having a frequency content wider than numerical signals, even though the general pattern of variation along the bridge was captured. The reasons for the lack-of-fit, beyond the unavailability of the values of important parameters, are to be traced to the description of the trajectory, to the sensitivity of the problem to pedestrians' inter-variability, and to the lack of intra-variability in the implementation of the model. This is probably the main cause of the discrepancy in terms of frequency range.

It can be concluded that the procedure proposed in this work establishes a reliable framework of analysis for the vertical footbridgepedestrian interaction. The new SMD model is a starting point for future developments, from the implementation of non-periodic gait to a more refined mechanical modelling. The formulation in Section 4 holds whatever the model complexity, since any pedestrian will have two contact points with the bridge at most. Thus, complexity remains confined to the development of the model and to the derivation of its equation of motion. Experimental tests are necessary to calibrate any mechanical model adopted for the pedestrian and, associated with numerical simulations, to identify the optimal values of the parameters. 


\section{Row synchronization}
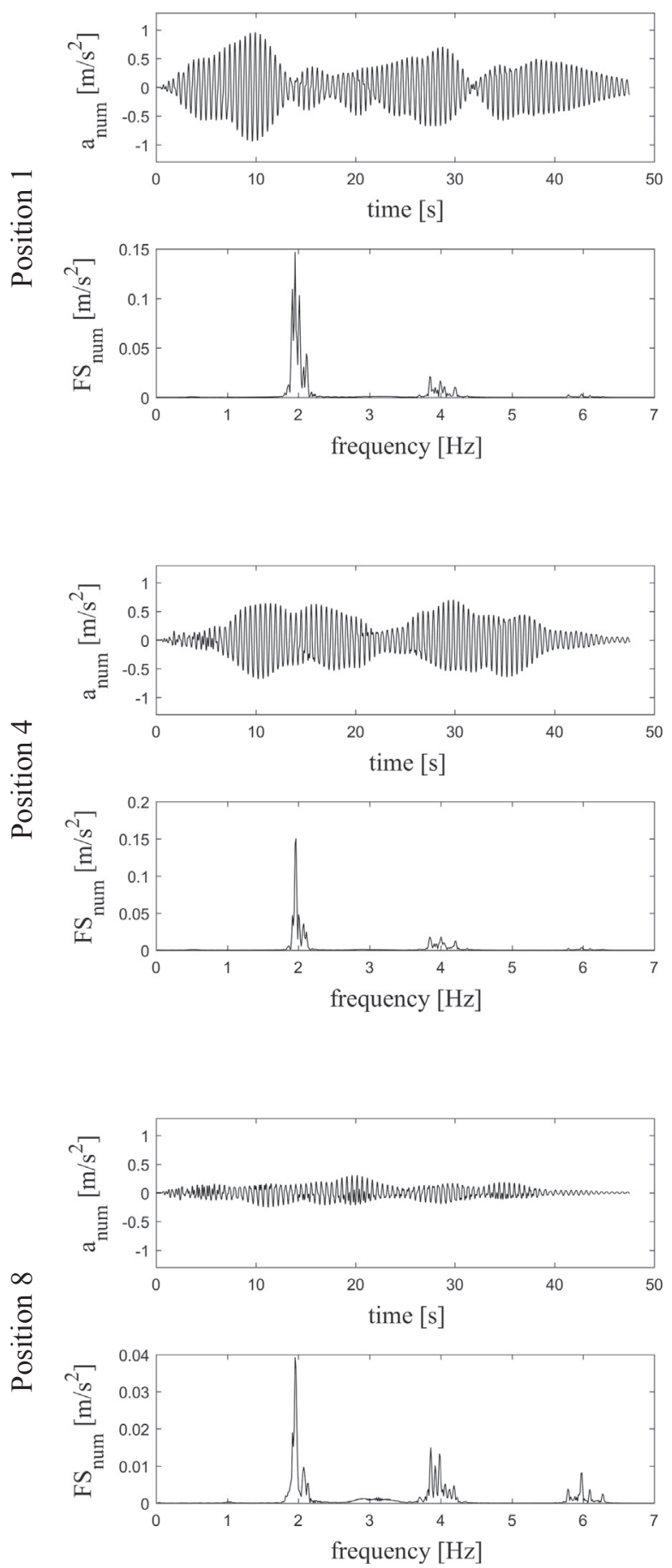

Row synchronization with phase difference
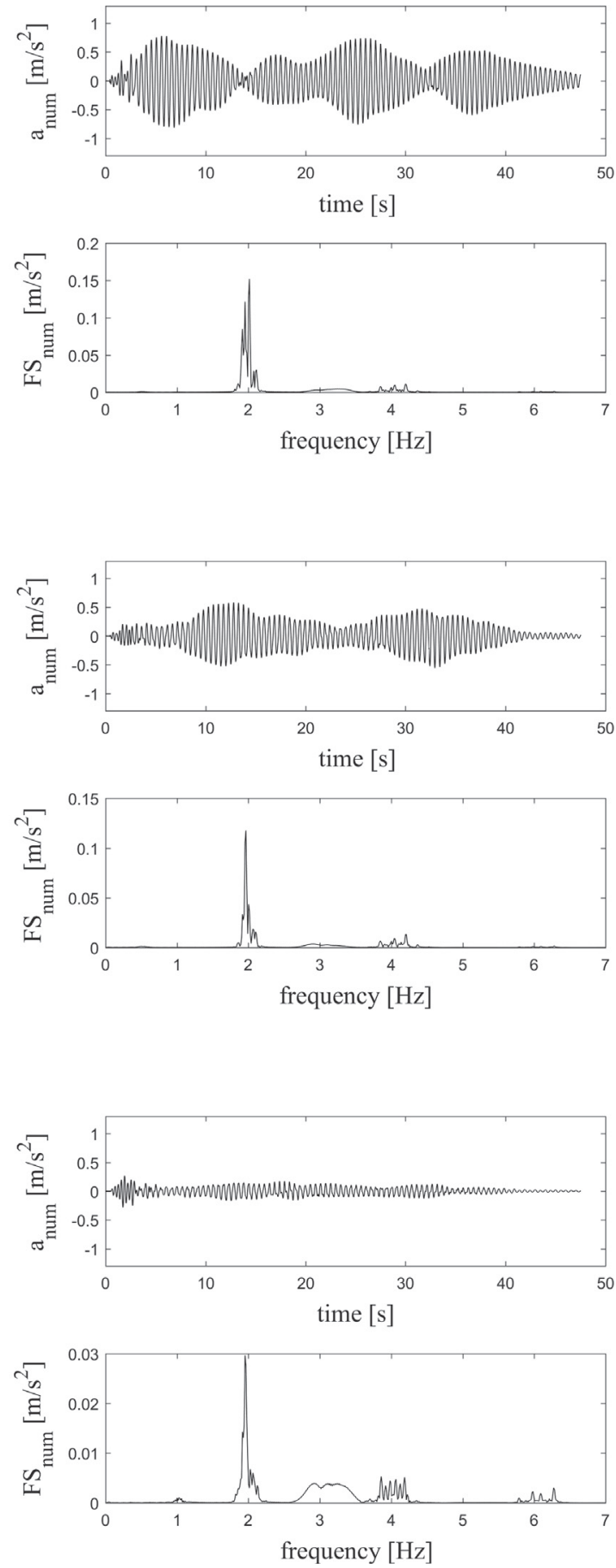

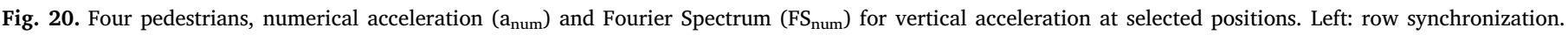
Right: row synchronization with phase difference.

From the engineering standpoint, the numerical simulations show the significant variability in the response due to relatively small variations in the loading scenarios. Finally, the procedure could rely on a commercial code for the solution of the structure. This advantage could be exploited if a different iteration strategy were adopted, on the whole time history and not at each time step [29].

\section{Acknowledgements}

The partial financial contribution of Italian MIUR (Ministry of
Higher Education and Research) under grant PRIN 2015-2018, Project 2015TTJN95, "Identification and monitoring of complex structural systems", is acknowledged. C. Gentile provided the experimental data. G. Palamà, MS in Civil Engineering, ran the analyses necessary for the data in Table 2 and Figs. 7 and 8. F. Perotti and V. Racic provided useful discussions on the final draft of the first version of the paper. Reviewers' comments helped improve the paper. All these contributions are gratefully acknowledged. 


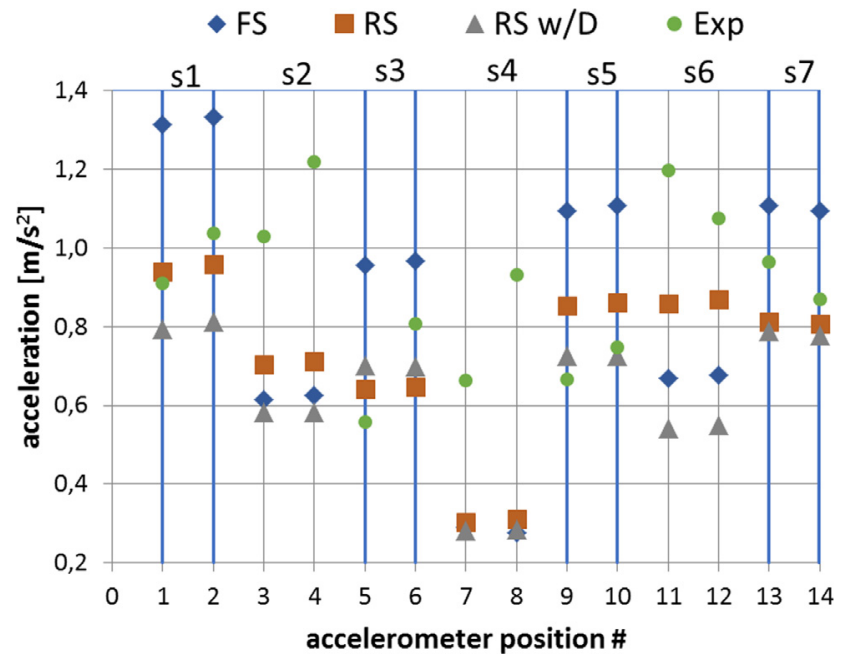

Fig. 21. Four pedestrians, peak values of acceleration at the accelerometers positions, for experimental and numerical results. FS: full synchronization, RS: row synchronization, RS w/D: row synchronization with delay. Thick lines delimit sections s1, s3, s5 and s7.

\section{References}

[1] Dallard P, Fitzpatrick A, Flint A, Bourva S, Low A, Ridsdill R, et al. The London Millenium footbridge. Struct Eng 2001;79(22):17-33.

[2] Ingólfsson ET, Georgakis CT, Jönsson J. Pedestrian-induced lateral vibrations of footbridges: a literature review. Eng Struct 2012;45:21-52.

[3] Schlaich M. Planning conditions for footbridges. In: Proceedings of the International Conference on the Design and Dynamic Behaviour of Footbridges, Paris, France, November 20-22, 40-52; 2002.

[4] Van Nimmen K, Lombaert G, De Roeck G, Van den Broeck P. Vibration serviceability of footbridges: evaluation of the current codes of practice. Eng Struct 2014;59:448-61.

[5] Živanović S. Benchmark footbridges for vibration serviceability assessment under the vertical component of pedestrian load. ASCE J Struct Eng 2012;193:1193-202.

[6] Zhang M, Georgakis C, Chen J. Biomechanically excited SMD model of a walking pedestrian. ASCE J Bridge Eng 2016;C4016003.

[7] Živanović S, Pavic A, Reynolds P. Vibration serviceability of footbridges under human-induced excitation: a literature review. J Sound Vib 2005;179:1-74.

[8] Zhang M, Georgakis C, Qu W, Chen J. SMD model parameters of pedestrians for vertical human-structure interaction. In: Proceedings of 33rd IMAC Conference and Exposition on Structural Dynamics, February, Orlando, USA; 2015.

[9] Sachse R, Pavic A, Reynolds P. Human-structure dynamics interaction in civil engineering dynamics: a literature review. Shock Vibration Digest 2003.

[10] Toso MA, Gomes HM, De Silva FT, Pimentel RL. Experimentally fitted biodynamic models for pedestrian-structure interaction in walking situations. J Mech Syst Signal Process 2016;72-72:590-606.

[11] Bocian M, MacDonald JHG, Burn JF. Biomechanically inspired modelling of pedestrian induced forces on lateral oscillating structures. J Sound Vib 2012;331:3914-29.

[12] Georgakis TC, Jorgensen GN. Change in mass and damping on vertically vibrating footbridges due to pedestrians. In: Proceedings of the 31st International Modal Analysis Conference on Structural Dynamics IMAC, February, Garden Grove, USA; 2013.

[13] Caprani CC, Ahmadi E. Formulation of human-structure interaction system models. J Sound Vib 2016;377:346-67.

[14] Hongli L, Zhengqing C. Analytical and experimental study on vertically dynamic interaction between human and bridge. China Civil Eng J 2014;47(6):78-87.

[15] Jimenez-Alonso JF, Sáez A, Caetano E, Magalhãe F. Vertical crowd-structure interaction model to analyze the change of the modal properties of a footbridge. ASCE J Bridge Eng 2016;C4015004.

[16] Shahabpoor E, Pavic A, Racic V. Structural vibration serviceability: new design framework featuring human-structure interaction. Eng Struct 2017;136:295-311.

[17] Kim SH, Mha HS, Cho KI, Won JH, Kim MA. Study on dynamic behaviour of footbridge under pedestrian excitation generated by human body model. In: Proceedings of the 8th Pacific Structural Steel Conference - Steel Structures in Natural Hazards PSSC, March, New Zealand; 2007.

[18] Da Silva FT, Pimentel RL. Biodynamic walking model for vibration serviceability of footbridges in vertical direction. In: Proceedings of the 8th International Conference on Structural Dynamics (EURODYN), July, Leuven, Belgium; 2011.

[19] Da Silva FT, Brito HMBF, Pimentel RL. Modelling of crowd load in vertical direction using biodynamic model for pedestrians crossing footbridges. Can J Civ Eng 2013;420(12):1196-204.

[20] Van Nimmen K, Maes K, Zivanovic S, Lombaert G, De Roeck G, Van de Broeck P. Identification and modelling of vertical human-structure interaction. In: Proceedings of 33rd IMAC Conference and Exposition on Structural Dynamics, February, Orlando, USA; 2015.

[21] Venuti F, Racic V, Corbetta A. Modelling framework for dynamic interaction between multiple pedestrians and vertical vibrations of footbridge. J Sound Vib 2016;379:245-63.

[22] Bocian M, Macdonald JHG, Burn JF. Biomechanically inspired modelling of pedestrian-induced vertical self-excited forces. ASCE J Bridge Eng 2013. https://doi. org/10.1061/(ASCE)BE.1943-5592.0000490, 1336-1346.

[23] Geyer H, Seyfarth A, Blickhan R. Compliant leg behaviour explains basic dynamics of walking and running. Proc. Biol. Sci. 2006;273(1603):2861-7.

[24] Kim S, Park S. Leg stiffness increases with speed to modulate gait frequency and propulsion energy. J Biomech 2011;44(7):1253-8.

[25] Qin JW, Law SS, Yang QS, Yang N. Pedestrian-bridge dynamic interaction, in cluding human participation. J Sound Vibr 2013;332(4):1107-24.

[26] Whittington BR, Thelen DG. A simple mass-spring model with roller feet can induce the ground reactions observed in human walking. J Biomech Eng 2009;131(1):011013.

[27] Gomez D, Silva CE, Dyke SJ, Thomson P. Interactive platform to include humanstructure interaction effects in the analysis of footbridges. In: Proceedings of 33rd IMAC Conference and Exposition on Structural Dynamics, February, Orlando, USA 2015.

[28] Research Fund for Coal and Steel. HiVoSS: Design of footbridges; 2008.

29] Feriani A, Mulas MG, Lucchini G. Vehicle-bridge dynamic interaction: an uncoupled approach. In: Proc. of International Conference on Noise and Vibration ISMA 2008 609-624, Paper ID 142, September, Leuven, Belgium; 2008.

[30] Bocian M, Brownjohn JMW, Racic V, Hester D, Quattrone A, Monnickendam R. A framework for experimental determination of localised vertical pedestrian forces on full-scale structures using wireless attitude and heading reference systems. J Sound Vib 2016;376:217-43.

[31] Van Nimmen K, Lombaert G, Jonkers I, De Roeck G, Van den Broek P. Characterisation of walking loads by 3D inertial motion tracking. J Sound Vib 2014;333:5212-26.

[32] Lai E, Gentile C, Mulas MG. Experimental and numerical assessment of a steel suspension footbridge. J Constr Steel Res 2017;132:16-28.

[33] Racic V, Pavic A, Brownjohn JMW. Experimental identification and analytical modeling of human walking forces: literature review. J Sound Vib 2009;326:1-49.

[34] Li Q, Fan J, Nie J, Li Q, Chen Y. Crowd-induced random vibration of footbridge and vibration control using multiple tuned mass dampers. J Sound Vib 2010;329:4068-92.

[35] Ebrahimpour A, Hamam A, Sack RL, Patten WN. Measuring and modelling dynamic loads imposed by moving crowds. J Struct Eng ASCE 1996;122(12):1468-74.

[36] Dougill JW, Wright JR, Parkhouse JG, Harrison RE. Human structure interaction during rhythmic bobbing. Struct Eng 2006;84(22):32-9.

[37] Pachi A, Ji T. Frequency and velocity of people walking. Struct Eng 2005;83:36-40.

[38] Van Nimmen K, Lombaert G, De Roeck G, Van den Broeck P. The impact of vertical human-structure interaction on the response of footbridges to pedestrian excitation. J Sound Vib 2017;402:104-21.

[39] Brincker R, Zhang LM, Andersen P. Modal identification from output-only systems using frequency domain decomposition. Smart Mater Struct 2001;10:441-5.

[40] Van Overschee P, De Moor B. Subspace Identification for Linear Systems: Theory, Implementation, Applications. Boston/London/Dordrecht: Kluwer; 1996.

[41] Lai E. Pedestrian-footbridge dynamic interaction: uncoupled analysis using a MSD model [PhD Thesis]. Politecnico di Milano, Department of Civil and Environmental Engineering; 2016.

[42] Lai E, Mulas MG. Pedestrian-footbridge dynamic interaction: a probabilistic assessment of vibration serviceability. Meccanica dei Materiali e delle Strutture 2016;VI(1):211-8.

[43] Hilber HM, Hughes TIR, Taylor RL. Improved numerical dissipation for time integration algorithms in structural dynamics. Earthq Eng Struct Dyn 1977;5:283-92.

[44] Fryba L. Vibration of Solids and Structures Under Moving Loads. Groningen: Noordhoff International Publishing; 1972.

[45] ANSYS. Online Manuals Release 5.5, < http://mostreal.sk/html/guide_55/ GBooktoc.html > ; 2015. 